\title{
Synthesis of the Sialic Acid (-)-KDN and Certain Epimers from (-)-3-Dehydroshikimic Acid or (-)-Quinic Acid
}

Martin G. Banwell, ${ }^{*}$ Natasha L. Hungerford and Katrina A. Jolliffe

Research School of Chemistry, Institute of Advanced Studies, The Australian National University, Canberra, ACT 0200, Australia mgb@rsc.anu.edu.au

\section{Contents}

General Experimental

Experimental Procedures and Product Characterization for Compounds: 5

$\begin{array}{rl}\mathbf{6} & \text { S4-S5 } \\ \mathbf{8} & \text { S5-S6 } \\ \mathbf{9} & \text { S6 } \\ \mathbf{1 1 - 1 6} & \mathrm{S} 7-\mathrm{S} 12 \\ 7-\text { epi-3 } & \mathrm{S} 12 \\ \mathbf{1 7} & \mathrm{S} 13-\mathrm{S} 14 \\ \text { 8-epi-3 } & \mathrm{S} 14 \\ \mathbf{1 8 - 1 9} & \mathrm{S} 15-\mathrm{S} 16\end{array}$

Allylic Alcohol Derived from Compound 19

20-21

References

${ }^{1} \mathrm{H}$ and ${ }^{13} \mathrm{C}$ NMR Spectra of Compounds: 6, 8, 9, 11-19,

Allylic Alcohol Derived from Compound 19 and 20-21
Page

S2

S17

S18-S20

S20

S21-S50 


\section{Experimental Procedures and Product Characterization}

\section{General Experimental:}

Melting points were measured on a Reichert hot-stage microscope apparatus and are uncorrected. Proton $\left({ }^{1} \mathrm{H}\right)$ and carbon $\left({ }^{13} \mathrm{C}\right) \mathrm{NMR}$ spectra were recorded on a Varian Inova 500 spectrometer operating at $500 \mathrm{MHz}$ for proton and $126 \mathrm{MHz}$ for carbon. In certain cases a Gemini 300 NMR spectrometer, operating at $300 \mathrm{MHz}$ (for proton) and $75 \mathrm{MHz}$ (for carbon), was employed. Spectra were acquired at $20{ }^{\circ} \mathrm{C}$ in deuterochloroform $\left(\mathrm{CDCl}_{3}\right)$ which had been filtered through basic alumina prior to use, or deuteromethanol $\left(\mathrm{CD}_{3} \mathrm{OD}\right)$ or deuterium oxide $\left(\mathrm{D}_{2} \mathrm{O}\right)$. Signals arising from the residual protio-forms of the solvent were used as the internal standard. Chemical shifts were recorded as $\delta$ values in parts per million (ppm). The assignments of signals observed in the various NMR spectra were often assisted by conducting DEPT, APT, homonuclear $\left({ }^{1} \mathrm{H} /{ }^{1} \mathrm{H}\right)$ correlation spectroscopy (gDQFCOSY), and/or heteronuclear $\left({ }^{1} \mathrm{H} /{ }^{13} \mathrm{C}\right)$ correlation spectroscopy (gHMQC or gHMBC) experiments. The term a-t refers to an apparent triplet. Infrared spectra $\left(\nu_{\max }\right)$ were recorded on a Perkin-Elmer 1800 Series FTIR Spectrometer. Samples were analysed as $\mathrm{KBr}$ disks (for solids) or as thin films on $\mathrm{NaCl}$ plates (for oils). Low resolution mass spectra were recorded on a Micromass-Waters LC-ZMD single quadrupole liquid chromatograph-MS or VG Quattro II triple quadrupole MS instrument using electrospray techniques in positive and/or negative ionization mode. Low resolution EI and FAB mass spectra were recorded on an AUTOSPEC spectrometer or a Kratos Analytical Concept ISQ instrument, the latter being located at the University of Tasmania. High resolution mass spectra were acquired by FAB methods on a Thermoquest Mat95XL instrument (located at CSIRO Molecular Science, Melbourne) or by EI methods on an AUTOSPEC instrument. Optical rotations were measured with a Perkin-Elmer 241 polarimeter at the sodium-D line $(589 \mathrm{~nm})$ and the concentrations $(c)(\mathrm{g} / 100 \mathrm{~mL})$ indicated using spectroscopic grade $\mathrm{CHCl}_{3}$ unless otherwise specified. The measurements were carried out in a cell with a path length (1) of $1 \mathrm{dm}$. Specific rotations $[\alpha]_{\mathrm{D}}$ (determined at the temperature indicated) were calculated using the equation $[\alpha]_{\mathrm{D}}=100 . \alpha /(c .1)$ and are given in $10^{-1} \cdot \mathrm{deg} \cdot \mathrm{cm}^{2} \cdot \mathrm{g}^{-1}$. Elemental analyses were performed by the Australian National University's Microanalytical Services Unit based at the Research School of Chemistry, Canberra, Australia. The unit cell parameters were recorded on a Nonius Kappa CCD instrument. Dichloromethane and chloroform were distilled from calcium hydride and THF and benzene were distilled, under nitrogen, from sodium benzophenone ketyl. Where necessary, reactions were performed under a nitrogen atmosphere. 


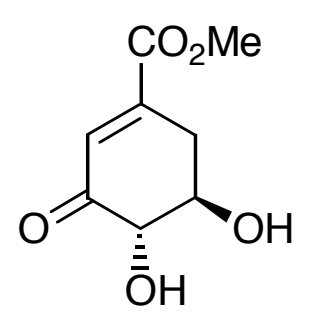

5

\begin{abstract}
A solution of diazomethane in diethyl ether was added dropwise to a solution of (-)-3-dehydroshikimic acid $4(3.0 \mathrm{~g}, 17 \mathrm{mmol})$ in $\mathrm{CH}_{3} \mathrm{OH}(20 \mathrm{~mL})$ at $-15^{\circ} \mathrm{C}$ until the yellow color remained. At this point a stream of nitrogen was blown over the reaction mixture to remove excess diazomethane then the solvent was removed under reduced pressure. The orange residue thus obtained was triturated with diethyl ether $/ \mathrm{CH}_{3} \mathrm{OH}(12 \mathrm{~mL}$ of a $5: 1 \mathrm{v} / \mathrm{v}$ mixture) to yield the methyl ester $5(2.5 \mathrm{~g}, 77 \%)$ as a colorless solid, mp 120-122 ${ }^{\circ} \mathrm{C}$ (lit. $\left.{ }^{1} \mathrm{mp} 124-125^{\circ} \mathrm{C}\right) ;{ }^{1} \mathrm{H}$ NMR $\left(300 \mathrm{MHz}, \mathrm{CD}_{3} \mathrm{OD}\right) \delta 6.71(1 \mathrm{H}, \mathrm{d}, \mathrm{H} 7$, J 3.1), 4.07 (1H, d, H5, J 10.5), 3.87 (1H, ddd, H4, J 10.5, 9.3, 5.1), 3.83 (3H, s, OMe), 3.09 (1H, dd, H3, $J$ 18.3, 5.1), 2.55 (1H, ddd, H3', $J$ 18.3, 9.3, 3.1 Hz); ${ }^{13} \mathrm{C}$ NMR (75 MHz, CD 3 OD) $\delta 200.6(\mathrm{C})$, $167.2(\mathrm{C}), 147.3(\mathrm{C}), 132.1(\mathrm{CH}), 80.1(\mathrm{CH}), 72.3(\mathrm{CH}), 53.3\left(\mathrm{CH}_{3}\right), 34.3\left(\mathrm{CH}_{2}\right)$.
\end{abstract}




\section{Compound 6}

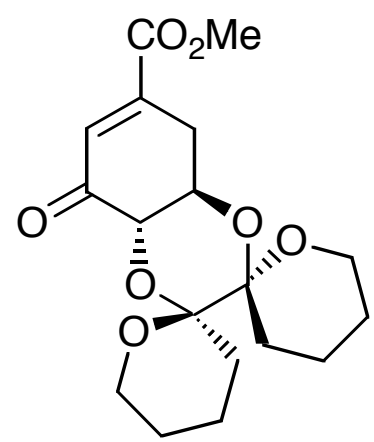

6

\section{From 5:}

1(S)-(+)-Camphor-10-sulfonic acid (110 mg, $0.47 \mathrm{mmol}$ ) was added to a solution of diol 5 (800 $\mathrm{mg}, 4.3$ $\mathrm{mmol})$ and bis-dihydropyran $(1.20 \mathrm{~g}, 7.2 \mathrm{mmol})$ in anhydrous $\mathrm{CHCl}_{3}(60 \mathrm{~mL})$ and the resulting mixture was heated at reflux for $48 \mathrm{~h}$. After cooling the resulting solution was diluted with $\mathrm{CH}_{2} \mathrm{Cl}_{2}(20 \mathrm{~mL})$, washed with $\mathrm{NaHCO}_{3}(3 \times 30 \mathrm{~mL}$ of a sat aq solution $)$ then dried $\left(\mathrm{MgSO}_{4}\right)$, filtered and concentrated under reduced pressure to give a brown oil. Subjection of this material to flash chromatography (1:3 $\mathrm{v} / \mathrm{v}$ EtOAc/hexane elution) and concentration of the appropriate fractions $\left(\mathrm{R}_{f} 0.3\right)$ gave a pale-yellow oil which was triturated with ice-cold EtOAc to provide the dispiroketal $6(1.20 \mathrm{~g}, 79 \%)$ as a colorless solid, mp $153-154{ }^{\circ} \mathrm{C} ;[\alpha]_{\mathrm{D}}^{18}+45.7\left(\mathrm{c} 1.0\right.$ in $\left.\mathrm{CHCl}_{3}\right)$.

\section{From 9:2}

Pyridinium chlorochromate $(858 \mathrm{mg}, 4.0 \mathrm{mmol})$ was added to a magnetically stirred mixture of compound 9 (370 mg, $0.99 \mathrm{mmol}), 4 \AA$ molecular sieves (powdered, $740 \mathrm{mg}$ ) and pyridine (323 mg, $0.33 \mathrm{~mL}, 4.08 \mathrm{mmol})$ in $\mathrm{CH}_{2} \mathrm{Cl}_{2}(4 \mathrm{~mL})$. The mixture was stirred at $18{ }^{\circ} \mathrm{C}$ for $24 \mathrm{~h}$ at which time TLC analysis (1:1 v/v EtOAc/hexane elution) showed an absence of starting material $\left(\mathrm{R}_{f} 0.2\right)$ and the formation of two products, $\mathbf{6}$ and $\mathbf{1 0}\left(\mathrm{R}_{f} 0.6\right.$ and $\mathrm{R}_{f} 0.3$, respectively). The reaction mixture was applied directly to a silica pad and eluted with EtOAc. The residue obtained upon concentration of the filtrate under reduced pressure was dissolved in $\mathrm{CH}_{2} \mathrm{Cl}_{2}(3 \mathrm{~mL})$ and the resulting solution cooled to $0{ }^{\circ} \mathrm{C}$, then treated with Hunig's base (384 mg, $0.517 \mathrm{~mL}, 2.97 \mathrm{mmol})$, DMAP (12 mg, $0.099 \mathrm{mmol}$ ) and acetic anhydride $(152 \mathrm{mg}, 0.140 \mathrm{~mL}, 1.49 \mathrm{mmol})$. The reaction mixture was stirred overnight at ca. $18{ }^{\circ} \mathrm{C}$, at which point TLC analysis (1:1 v/v EtOAc/hexane elution) indicated complete conversion of the more polar product $\left(\mathrm{R}_{f} 0.3\right)$ to the less polar one $\left(\mathrm{R}_{f} 0.6\right)$. Silica gel $(0.5 \mathrm{~g})$ was added to the reaction mixture which was then concentrated under reduced pressure to yield a pale-yellow powder. This was subjected to flash chromatography (short silica gel pad; 1:1 v/v EtOAc/hexane elution). Concentration of the appropriate fractions $\left(\mathrm{R}_{f} 0.6\right)$ yielded compound $\mathbf{6},(235 \mathrm{mg}, 67 \%)$ as a colorless solid, $\mathrm{mp} 153-154^{\circ} \mathrm{C}$; 
$[\alpha]_{\mathrm{D}}^{18}+49.0\left(c 0.4, \mathrm{CHCl}_{3}\right) ;{ }^{1} \mathrm{H} \mathrm{NMR}\left(500 \mathrm{MHz}, \mathrm{CDCl}_{3}\right) \delta 6.81(1 \mathrm{H}, \mathrm{d}, \mathrm{H} 7, J 3.4), 4.34(1 \mathrm{H}, \mathrm{d}, \mathrm{H} 5, J$ 11.2), $4.16\left(1 \mathrm{H}\right.$, ddd, $\mathrm{H} 4, J$ 11.2, 10.7, 5.4), $3.85(3 \mathrm{H}, \mathrm{s}, \mathrm{OMe}), 3.71-3.59\left(4 \mathrm{H}\right.$, complex m, $\left.2 \times \mathrm{CH}_{2}\right)$, 3.10 (1H, dd, H3, J 18.3, 5.4), 2.66 (1H, ddd, H3, J 18.3, 10.7, 3.4 Hz), 2.05-1.73 (4H, complex m, $2 \times$ $\left.\mathrm{CH}_{2}\right), 1.66-1.40\left(8 \mathrm{H}\right.$, complex m, $\left.4 \times \mathrm{CH}_{2}\right) ;{ }^{13} \mathrm{C} \mathrm{NMR}\left(75 \mathrm{MHz}, \mathrm{CDCl}_{3}\right) \delta 194.7$ (C), $166.0(\mathrm{C}), 144.5$ (C), $132.8(\mathrm{CH}), 97.5(\mathrm{C}), 96.6(\mathrm{C}), 74.2(\mathrm{CH}), 66.4(\mathrm{CH}), 61.1\left(\mathrm{CH}_{2}\right), 60.8\left(\mathrm{CH}_{2}\right), 52.9\left(\mathrm{CH}_{3}\right), 30.4$ $\left(\mathrm{CH}_{2}\right), 28.3(3)\left(\mathrm{CH}_{2}\right), 28.2(5)\left(\mathrm{CH}_{2}\right), 24.8\left(\mathrm{CH}_{2}\right.$, two signals superimposed), 17.9(3) $\left(\mathrm{CH}_{2}\right), 17.8(8)$ $\left(\mathrm{CH}_{2}\right)$; IR (NaCl, thin film) 2951, 2874, 1727, 1707, 1639, 1439, 1381, 1356, 1249, 1211, 1191, 1159, 1143, 1098, 1073, 1045, 994, 883, $736 \mathrm{~cm}^{-1}$; MS (ESI+) m/z $375\left(\mathrm{M}+\mathrm{Na}^{+}, 100 \%\right)$; Anal. Calcd for $\mathrm{C}_{18} \mathrm{H}_{24} \mathrm{O}_{7}: \mathrm{C} 61.35$, H 6.86. Found: C 60.90, H 7.15\%.

\section{Compound 8}

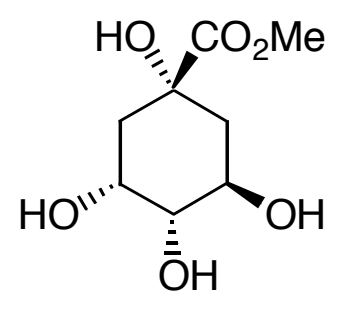

8

Methanolic $\mathrm{HCl}$ was generated by the dropwise addition of acetyl chloride $(1.75 \mathrm{~g}, 1.6 \mathrm{~mL}, 22.3 \mathrm{mmol})$ to $\mathrm{CH}_{3} \mathrm{OH}(35 \mathrm{~mL})$ at $0^{\circ} \mathrm{C}$. The resulting solution was transferred to a flask containing quinic acid (7) $(2.00 \mathrm{~g}, 10.4 \mathrm{mmol})$ and the reaction mixture was stirred at reflux overnight. TLC analysis $(1: 7 \mathrm{v} / \mathrm{v}$ $\mathrm{CH}_{3} \mathrm{OH} / \mathrm{EtOAc}$ elution) after this time showed complete conversion of starting material $\left(\mathrm{R}_{f} 0.0\right)$ to product $\left(\mathrm{R}_{f} 0.2\right)$. As a result, the reaction mixture was filtered through a pad of Celite ${ }^{\mathrm{TM}}$ which was washed with $\mathrm{CH}_{3} \mathrm{OH}(50 \mathrm{~mL})$. Concentration of the combined filtrates under reduced pressure yielded a residue which was subjected to flash chromatography (short silica gel pad; 1:7 v/v $\mathrm{CH}_{3} \mathrm{OH} / \mathrm{EtOAc}$ elution). Concentration of the appropriate fractions $\left(\mathrm{R}_{f} 0.2\right)$ yielded compound $\mathbf{8}^{\mathbf{3}}(1.92 \mathrm{~g}, 90 \%)$ as colorless needles, mp $126-127^{\circ} \mathrm{C}\left(\right.$ lit. $\left.^{3} \mathrm{mp} 118^{\circ} \mathrm{C}\right) ;[\alpha]_{\mathrm{D}}^{19}-30.9\left(c 1.86, \mathrm{CH}_{3} \mathrm{OH}\right)\left\{\right.$ lit. $^{4}[\alpha]_{\mathrm{D}}^{20}-31.6(c$ 1.45, $\left.\left.\mathrm{CH}_{3} \mathrm{OH}\right)\right\} ;{ }^{1} \mathrm{H} \mathrm{NMR}\left(500 \mathrm{MHz}, \mathrm{CD}_{3} \mathrm{OD}\right) \delta 4.07$ (1H, m, H6), 3.98 (1H, ddd, $\mathrm{H} 4, J$ 10.0, 8.5, 4.4), $3.72(3 \mathrm{H}, \mathrm{s}, \mathrm{OMe}), 3.40\left(1 \mathrm{H}, \mathrm{dd}, \mathrm{H} 5, J\right.$ 8.5, 2.8), $2.09\left(1 \mathrm{H}, \mathrm{ddd}, \mathrm{H}_{\text {eq }}, J 13.2,4.4,2.2\right), 2.06(1 \mathrm{H}, \mathrm{dd}$, $\left.\mathrm{H}_{\text {ax }}, J 14.2,3.2\right), 1.99\left(1 \mathrm{H}, \mathrm{ddd}, \mathrm{H} 7_{\text {eq }}, J 14.2,4.4,2.2\right), 1.86\left(1 \mathrm{H}, \mathrm{dd}, \mathrm{H}_{\text {ax }}, J 13.2,10.0 \mathrm{~Hz}\right) ;{ }^{13} \mathrm{C} \mathrm{NMR}$ (125.7 MHz, $\left.\mathrm{CD}_{3} \mathrm{OD}\right) \delta 175.9(\mathrm{C}), 76.8(\mathrm{C}), 76.5(\mathrm{CH}), 71.3(\mathrm{CH}), 68.1(\mathrm{CH}), 52.9\left(\mathrm{CH}_{3}\right), 41.9\left(\mathrm{CH}_{2}\right)$, 
$38.2\left(\mathrm{CH}_{2}\right)$; IR (NaCl, thin film) 3340, 1734, 1435, 1245, 1122, $1072 \mathrm{~cm}^{-1} ; \mathrm{MS}$ (ESI+) $\mathrm{m} / z$ $\left(2 \mathrm{M}+\mathrm{Na}^{+}, 5 \%\right), 413\left(2 \mathrm{M}+\mathrm{H}^{+}, 5\right), 229\left(\mathrm{M}+\mathrm{Na}^{+}, 100\right)$; Anal. Calcd for $\mathrm{C}_{8} \mathrm{H}_{14} \mathrm{O}_{6}: \mathrm{C}, 46.60 ; \mathrm{H}, 6.84$. Found: C, 46.05; H, 7.24\%.

\section{Compound 9}

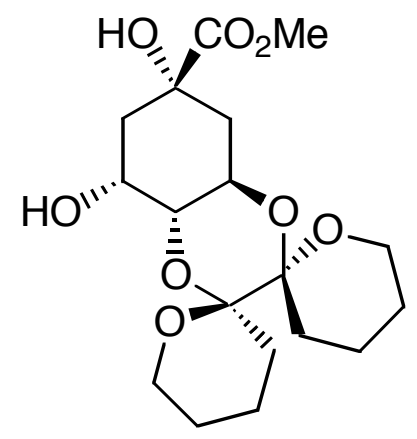

9

1(S)-(+)-Camphor-10-sulfonic acid (44 $\mathrm{mg}, 0.191 \mathrm{mmol}$ ) was added to a magnetically stirred solution of methyl quinate 8 (0.392 g, $1.91 \mathrm{mmol})$ and 2,2'-bis-dihydropyran (2,2'-bis-DHP) (475 mg, 2.86 $\mathrm{mmol})$ in dry $\mathrm{CHCl}_{3}(25 \mathrm{~mL})$ and the resulting mixture was heated at reflux for $16 \mathrm{~h}$. TLC analysis $(1: 1$ $\mathrm{v} / \mathrm{v}$ EtOAc/hexane elution) after this time revealed a mixture of products, with the major one appearing at $\mathrm{R}_{f}$ 0.2. The cooled reaction mixture was neutralized by the addition of $\mathrm{Et}_{3} \mathrm{~N}(100 \mu \mathrm{L})$ then concentrated under reduced pressure. The residue thus obtained was dissolved in $\mathrm{CHCl}_{3}$, preadsorbed onto silica and subjected to flash chromatography (1:2 then 1:1 v/v EtOAc/hexane elution). Concentration of the appropriate fractions $\left(\mathrm{R}_{f} 0.2 \mathrm{in} 1: 1 \mathrm{v} / \mathrm{v}\right.$ EtOAc/hexane) gave diol $9(407 \mathrm{mg}, 57 \%)$ as a colorless solid, mp $61-63{ }^{\circ} \mathrm{C} ;[\alpha]_{\mathrm{D}}^{19}+75.1\left(\mathrm{c} 1.07, \mathrm{CHCl}_{3}\right) ;{ }^{1} \mathrm{H}$ NMR $\left(500 \mathrm{MHz}, \mathrm{CDCl}_{3}\right) \delta 4.35$ (1H, ddd, H4, J 12.2, 10.0, 4.6), 4.22 (1H, dd, H6, J 5.9, 2.9), 3.78 (3H, s, OMe), 3.68-3.63 (5H, complex m, H5, $\left.2 \times \mathrm{CH}_{2}-\mathrm{O}\right), 2.20$ (1H, a-dt, $\left.\mathrm{H}_{\text {eq }}, J 15.1,2.9\right), 2.14$ (1H, ddd, $\left.\mathrm{H}_{\text {eq }}, J 12.7,4.4,2.9\right)$, $2.05\left(1 \mathrm{H}, \mathrm{dd}, \mathrm{H}_{\mathrm{ax}}, J 15.1,2.9\right), 1.94\left(1 \mathrm{H}, \mathrm{a}-\mathrm{t}, \mathrm{H} 3_{\mathrm{ax}}, J 12.2 \mathrm{~Hz}\right) 1.86-1.70\left(4 \mathrm{H}\right.$, complex m, $\left.2 \times \mathrm{CH}_{2}\right)$, 1.62-1.45 (8H, complex m, $\left.4 \times \mathrm{CH}_{2}\right) ;{ }^{13} \mathrm{C}$ NMR $\left(125.7 \mathrm{MHz}, \mathrm{CDCl}_{3}\right) \delta 174.3(\mathrm{C}), 97.6(\mathrm{C}), 75.8(\mathrm{C})$, $71.9(\mathrm{CH}), 69.3(\mathrm{CH}), 61.6(\mathrm{CH}), 60.7\left(\mathrm{CH}_{2}\right), 60.6\left(\mathrm{CH}_{2}\right), 52.9\left(\mathrm{CH}_{3}\right), 38.7\left(\mathrm{CH}_{2}\right), 37.2\left(\mathrm{CH}_{2}\right), 28.6$ $\left(\mathrm{CH}_{2}\right), 28.3\left(\mathrm{CH}_{2}\right), 24.9\left(\mathrm{CH}_{2}\right), 24.8\left(\mathrm{CH}_{2}\right), 18.0\left(\mathrm{CH}_{2}\right.$, two signals superimposed); IR ( $\mathrm{NaCl}$, thin film) 3468, 2947, 2873, 1735, 1437, 1352, 1240, 1210, 1161, 1136, 1072, 1044, 992, 972, $938 \mathrm{~cm}^{-1}$; MS $\left(\right.$ ESI+) $\mathrm{m} / z .767\left(2 \mathrm{M}+\mathrm{Na}^{+}, 25 \%\right), 395\left(\mathrm{M}+\mathrm{Na}^{+}, 100\right), 167$ (10); Anal. Calcd for $\mathrm{C}_{18} \mathrm{H}_{28} \mathrm{O}_{8}: \mathrm{C}, 58.05 ; \mathrm{H}$, 7.58. Found: C, 58.22; H, 7.72\%. 


\section{Alcohol 11}

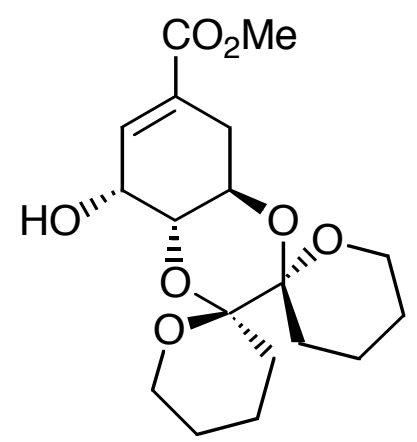

11

K-Selectride $(0.90 \mathrm{~mL}$ of a $1 \mathrm{M}$ solution in THF, $0.9 \mathrm{mmol})$ was added to a solution of enone 6 (263 $\mathrm{mg}, 0.75 \mathrm{mmol})$ in THF (6 mL) maintained at $-78{ }^{\circ} \mathrm{C}$ under an atmosphere of nitrogen and the resulting mixture was stirred at $-78^{\circ} \mathrm{C}$ for $20 \mathrm{~min}$. TLC analysis $(1: 2 \mathrm{v} / \mathrm{v}$ EtOAc/hexane elution $)$ after this time showed residual staring material $\left(\mathrm{R}_{f} 0.4\right)$ and a new product $\left(\mathrm{R}_{f} 0.3\right)$. Additional K-Selectride $(0.5 \mathrm{~mL}$ of a $1 \mathrm{M}$ solution in THF, $0.5 \mathrm{mmol})$ was added and after $20 \mathrm{~min}$ TLC analysis $(1: 2 \mathrm{v} / \mathrm{v}$ EtOAc/hexane elution) showed complete conversion of the starting enone to product $\left(\mathrm{R}_{f} 0.3\right)$. $\mathrm{NH}_{4} \mathrm{Cl}$ (10 $\mathrm{mL}$ of a sat aq solution) was then added and the mixture was warmed to ambient temperature. The mixture was partitioned between diethyl ether $(30 \mathrm{~mL})$ and $\mathrm{H}_{2} \mathrm{O}(30 \mathrm{~mL})$. The separated aqueous phase was extracted with diethyl ether $(2 \times 20 \mathrm{~mL})$ and the combined organic phases were dried $\left(\mathrm{Na}_{2} \mathrm{SO}_{4}\right)$, filtered and concentrated under reduced pressure to give a yellow oil. This material was dissolved in $\mathrm{CH}_{3} \mathrm{OH}(12 \mathrm{~mL})$ and silica gel $(2.5 \mathrm{~g})$ was added. The resulting mixture was stirred for $16 \mathrm{~h}$ at $18{ }^{\circ} \mathrm{C}$ and then the solvent was removed, under reduced pressure, to give the product adsorbed onto silica. Subjection of this material to flash chromatography (1:2 v/v EtOAc/hexane elution) and concentration of the appropriate fractions $\left(\mathrm{R}_{f} 0.3\right)$ gave the alcohol $11(227 \mathrm{mg}, 86 \%)$ as a colorless foam, $[\alpha]_{\mathrm{D}}-0.9$ (c 1.2 in $\left.\mathrm{CHCl}_{3}\right) ;{ }^{1} \mathrm{H}$ NMR $\left(300 \mathrm{MHz}, \mathrm{CDCl}_{3}\right) \delta 6.89(1 \mathrm{H}, \mathrm{dd}, \mathrm{H} 7, J$ 5.1, 2.5), $4.38(1 \mathrm{H}$, a-t, H6, J 4.8), 4.13 (1H, a-dt, H4, J 10.5, 5.9), 3.72 (3H, s, OMe), 3.70-3.55 (5H, complex m, H5, $2 \times \mathrm{CH}_{2}-\mathrm{O}$ ), 2.82 (1H, dd, H3, J 17.6, 5.9), 2.23 (1H, ddd, H3', J 17.6, 10.4, 2.8 Hz), 1.83-1.62 (4H, complex m, $2 \times$ $\left.\mathrm{CH}_{2}\right), 1.61-1.40\left(8 \mathrm{H}\right.$, complex m, $\left.4 \times \mathrm{CH}_{2}\right) ;{ }^{13} \mathrm{C} \mathrm{NMR}\left(75 \mathrm{MHz}, \mathrm{CDCl}_{3}\right) \delta 166.6(\mathrm{C}), 135.1(\mathrm{CH})$, 131.6 (C), $97.2(\mathrm{C}), 96.5(\mathrm{C}), 69.5(\mathrm{CH}), 64.9(\mathrm{CH}), 61.5(\mathrm{CH}), 60.8\left(\mathrm{CH}_{2}\right), 60.6\left(\mathrm{CH}_{2}\right), 52.0\left(\mathrm{CH}_{3}\right)$, $29.9\left(\mathrm{CH}_{2}\right), 28.5\left(\mathrm{CH}_{2}\right), 28.3\left(\mathrm{CH}_{2}\right), 24.9\left(\mathrm{CH}_{2}\right), 24.8\left(\mathrm{CH}_{2}\right), 17.9\left(\mathrm{CH}_{2}\right.$, two signals superimposed); IR (KBr) 3476, 2949, 2872, 1720, 1650, 1438, 1258, 1191, 1162, 1090, 1072, 1044, 994, $735 \mathrm{~cm}^{-1}$; MS 
(EI) $m / z 354\left(\mathrm{M}^{+\bullet}, 12 \%\right), 323$ (1), 296 (1), 254 (10), 225 (8), 167 (17), 154 (57), 101 (100), 95 (32), 83 (31); HRMS (EI) $m / z$ calcd for $\mathrm{C}_{18} \mathrm{H}_{26} \mathrm{O}_{7} \mathrm{M}^{+\bullet}: 354.1679$. Found: 354.1676.

\section{Aldehyde 12}

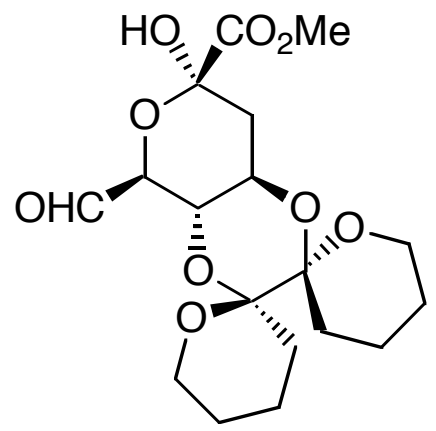

12

A stream of ozone in oxygen was bubbled through a solution of the alcohol 11 (227 $\mathrm{mg}, 0.64 \mathrm{mmol})$ in $\mathrm{CH}_{2} \mathrm{Cl}_{2}(30 \mathrm{~mL})$ maintained at $-78^{\circ} \mathrm{C}$ until the solution turned blue $(\sim 0.25 \mathrm{~h})$. TLC analysis $(2: 1 \mathrm{v} / \mathrm{v}$ EtOAc/hexane elution) then showed an absence of starting alcohol $\left(\mathrm{R}_{f} 0.6\right)$ so the solution was purged with a stream of nitrogen then $\mathrm{Me}_{2} \mathrm{~S}(0.50 \mathrm{~mL},>10$ eq.) was added, together with silica gel $(6 \mathrm{~g})$. The resulting mixture was stirred at $18^{\circ} \mathrm{C}$ for $16 \mathrm{~h}$ then concentrated under reduced pressure. The mixture thus obtained was subjected to rapid flash chromatography (short silica gel pad, 2:1 v/v EtOAc/hexane elution) to give, upon concentration of the appropriate fractions $\left(R_{\mathrm{f}} 0.14\right)$, the aldehyde $12(155 \mathrm{mg}$, $63 \%)$ as a colorless foam, $[\alpha]_{\mathrm{D}}^{21}+100.9\left(c 0.81, \mathrm{CHCl}_{3}\right) ;{ }^{1} \mathrm{H} \mathrm{NMR}\left(300 \mathrm{MHz}, \mathrm{CDCl}_{3}\right) \delta 9.74(1 \mathrm{H}, \mathrm{d}$, H7, J 0.9), 4.51 (1H, dd, H6, J 10.8, 0.9), 4.32 (1H, ddd, H4, J 12.3, 9.6, 4.8), 4.07 (1H, d, OH, J 1.8), $3.86(3 \mathrm{H}, \mathrm{s}, \mathrm{OMe}), 3.83(1 \mathrm{H}, \mathrm{m}, \mathrm{H} 5), 3.74-3.65\left(3 \mathrm{H}\right.$, complex m, $\left.3 \times \mathrm{CH}_{2}-\mathrm{O}\right), 3.54(1 \mathrm{H}, \mathrm{m}, 1 \times$ $\left.\mathrm{CH}_{2}-\mathrm{O}\right), 2.27$ (1H, ddd, H3, J 12.6, 12.3, 2.1), 2.06 (1H, dd, H3, J 12.6, 4.8 Hz), 1.85-1.41 (12H, complex m, $\left.6 \times \mathrm{CH}_{2}\right) ;{ }^{13} \mathrm{C}$ NMR $\left(75 \mathrm{MHz}, \mathrm{CDCl}_{3}\right) \delta 196.3(\mathrm{CH}), 169.3(\mathrm{C}), 97.6(\mathrm{C}), 97.4(\mathrm{C}), 95.5$ (C), $75.1(\mathrm{CH}), 68.1(\mathrm{CH}), 64.2(\mathrm{CH}), 61.1\left(\mathrm{CH}_{2}\right), 60.9\left(\mathrm{CH}_{2}\right), 53.8\left(\mathrm{CH}_{3}\right), 35.0\left(\mathrm{CH}_{2}\right), 28.6\left(\mathrm{CH}_{2}\right), 28.5$ $\left(\mathrm{CH}_{2}\right), 24.9\left(\mathrm{CH}_{2}\right), 24.8\left(\mathrm{CH}_{2}\right), 18.1\left(\mathrm{CH}_{2}\right), 18.0\left(\mathrm{CH}_{2}\right)$; IR $(\mathrm{KBr}) 3443,2948,2873,1744,1439,1273$, 1209, 1191, 1150, 1070, 1047, 988, 966, 899, $736 \mathrm{~cm}^{-1}$; MS (ESI+) m/z 795 (2M+Na+, 10\%), 468 $\left(\mathrm{M}+2 \mathrm{CH}_{3} \mathrm{CN}^{+}, 32\right), 450\left(\mathrm{M}+\mathrm{Na}^{+}+\mathrm{CH}_{3} \mathrm{CN}, 25\right), 427\left(\mathrm{M}+\mathrm{CH}_{3} \mathrm{CN}^{+}, 55\right), 409\left(\mathrm{M}+\mathrm{Na}^{+}, 27\right), 167(100)$; Anal. Calcd for $\mathrm{C}_{18} \mathrm{H}_{26} \mathrm{O}_{9}$ : C, 55.95; H, 6.78. Found: C, 56.20; H, 7.04\%. 


\section{Aldehyde 13}

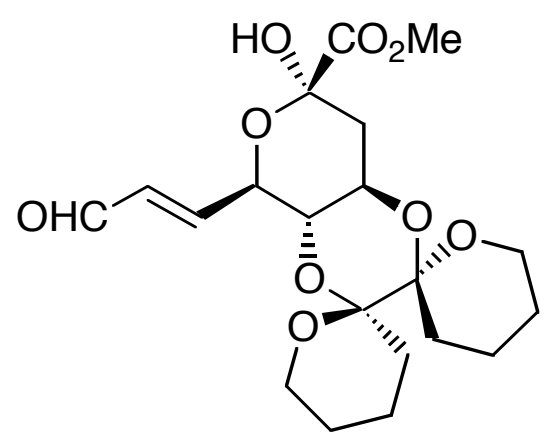

13

The aldehyde 12 (155 mg, $0.40 \mathrm{mmol}$ ) was dissolved in $\mathrm{CH}_{2} \mathrm{Cl}_{2}$ (13 mL) containing AcOH (3 drops) and then (triphenylphosphoranylidene)acetaldehyde $(128 \mathrm{mg}, 0.42 \mathrm{mmol}$ ) was added. The resulting orange solution was stirred at $18{ }^{\circ} \mathrm{C}$ for $18 \mathrm{~h}$ after which time TLC analysis $(2: 1 \mathrm{v} / \mathrm{v}$ EtOAc/hexane elution) showed the formation of a new product $\left(\mathrm{R}_{f} 0.7\right)$. Silica gel $(0.3 \mathrm{~g})$ was added and the solvent then removed under reduced pressure. The residue thus obtained was subjected to flash chromatography $\left(1: 1 \mathrm{v} / \mathrm{v}\right.$ EtOAc/hexane with $0.1 \% \mathrm{Et}_{3} \mathrm{~N}$ elution) to give the aldehyde 13 (128 mg, $78 \%$ ) as a colorless foam $\left(\mathrm{R}_{f} 0.6\right),[\alpha]_{\mathrm{D}}+137.7$ (c 1.2 in $\left.\mathrm{CHCl}_{3}\right) ;{ }^{1} \mathrm{H} \mathrm{NMR}\left(300 \mathrm{MHz}, \mathrm{CDCl}_{3}\right) \delta 9.57$ (1H, d, H9, J 8.1), 6.90 (1H, dd, H7, J 15.9, 4.2), 6.39 (1H, ddd, H8, J 15.9, 8.1, 1.5), 4.74 (1H, ddd, H6, J 10.2, 4.2, 1.5), 4.29 (1H, ddd, H4, J 12.3, 9.3, 4.8), 4.12 (1H, m, OH), 3.84 (3H, s, OMe), 3.74-3.61 (3H, complex m), 3.52 (1H, a-t, H5, J 9.9), 3.45 (1H, m), 2.27 (1H, a-dt, H3, J 12.3, 2.1), $2.07(1 \mathrm{H}, \mathrm{dd}, \mathrm{H} 3, J 12.3,4.8 \mathrm{~Hz}), 1.85-1.45\left(12 \mathrm{H}\right.$, complex m, $\left.6 \times \mathrm{CH}_{2}\right) ;{ }^{13} \mathrm{C} \mathrm{NMR}\left(75 \mathrm{MHz}, \mathrm{CDCl}_{3}\right) \delta$ $193.1(\mathrm{CH}), 169.5(\mathrm{C}), 151.4(\mathrm{CH}), 132.2(\mathrm{CH}), 97.6(\mathrm{C}), 97.2(\mathrm{C}), 95.4(\mathrm{C}), 71.0(\mathrm{CH}), 70.6(\mathrm{CH})$, $64.4(\mathrm{CH}), 60.9\left(\mathrm{CH}_{2}\right.$, two signals superimposed), $53.6\left(\mathrm{CH}_{3}\right), 35.2\left(\mathrm{CH}_{2}\right), 28.6\left(\mathrm{CH}_{2}\right), 28.5\left(\mathrm{CH}_{2}\right), 24.9$ $\left(\mathrm{CH}_{2}\right), 24.8\left(\mathrm{CH}_{2}\right), 18.2\left(\mathrm{CH}_{2}\right), 18.0\left(\mathrm{CH}_{2}\right)$; IR $(\mathrm{KBr})$ 3369, 2948, 2872, 1752, 1690, 1439, 1272, 1209, 1191, 1151, 1071, 1046, 989, 935, $899 \mathrm{~cm}^{-1}$; MS (EI) m/z $412\left(\mathrm{M}^{+\bullet}, 1 \%\right), 353$ (2), 312 (2), 283 (9), 265 (13), 212 (18), 194 (14), 168 (39), 140 (47), 101 (100); HRMS (EI) m/z calcd for $\mathrm{C}_{20} \mathrm{H}_{28} \mathrm{O}_{9} \mathrm{M}^{+\bullet}$ : 412.1733. Found: 412.1737. 


\section{Allylic alcohol 14}

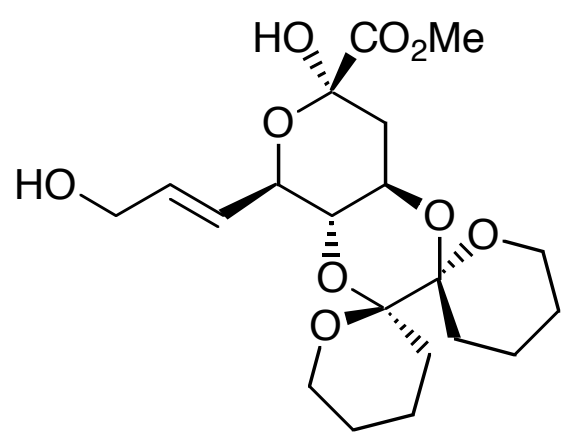

14

$\mathrm{CeCl}_{3} \cdot 7 \mathrm{H}_{2} \mathrm{O}(120 \mathrm{mg}, 0.323 \mathrm{mmol})$ was added to a solution of aldehyde $\mathbf{1 3}(121 \mathrm{mg}, 0.294 \mathrm{mmol})$ and 2,6-lutidine (157 mg, $171 \mu \mathrm{L}, 1.47 \mathrm{mmol})$ in $\mathrm{CH}_{2} \mathrm{Cl}_{2} / \mathrm{EtOH}(5.4 \mathrm{~mL}$ of a $1: 1 \mathrm{v} / \mathrm{v}$ mixture) maintained at ca. $18^{\circ} \mathrm{C}$. This mixture was then cooled to $-78^{\circ} \mathrm{C}$, treated with $\mathrm{NaBH}_{4}(33 \mathrm{mg}, 0.881 \mathrm{mmol})$ in EtOH $(0.87 \mathrm{~mL})$ and stirring continued at $-78^{\circ} \mathrm{C}$ for $1 \mathrm{~h}$. TLC analysis $(1: 2 \mathrm{v} / \mathrm{v}$ EtOAc/hexane elution) after this time showed the absence of starting material $\left(R_{f} 0.3\right)$ and the presence of a new product $\left(R_{f} 0.07\right)$. As a result acetone $(0.8 \mathrm{~mL})$ was added, the cooling bath was removed and the reaction mixture was poured into $\mathrm{H}_{2} \mathrm{O}(40 \mathrm{~mL}) . \mathrm{HCl}(5 \mathrm{~mL}$ of a $1 \mathrm{M}$ aq solution) was then added and the resulting mixture was extracted with $\mathrm{CH}_{2} \mathrm{Cl}_{2}(3 \times 20 \mathrm{~mL})$. The combined organic phases were washed with $\mathrm{NaCl}(15 \mathrm{~mL}$ of a sat aq solution) then dried $\left(\mathrm{Na}_{2} \mathrm{SO}_{4}\right)$, filtered and concentrated under reduced pressure to give a yellow oil. Subjection of this material to flash chromatography (1:1 v/v EtOAc/hexane with $0.5 \% \mathrm{NH}_{3}$ elution) and concentration of the appropriate fractions $\left(\mathrm{R}_{f} 0.2\right)$ gave allylic alcohol $14(64 \mathrm{mg}, 50 \%)$ as a colorless foam, $[\alpha]_{\mathrm{D}}+128.6\left(c 0.7\right.$ in $\left.\mathrm{CHCl}_{3}\right) ;{ }^{1} \mathrm{H} \mathrm{NMR}\left(300 \mathrm{MHz}, \mathrm{CDCl}_{3}\right) \delta 6.03(1 \mathrm{H}, \mathrm{dtd}, \mathrm{H} 8, J$ 15.5, 5.4, 1.1), 5.77 (1H, tdd, H7, J 15.5, 6.3, 1.5), 4.47 (1H, ddd, H6, J 10.0, 6.3, 0.9), 4.23 (1H, ddd, H4, J 12.2, 9.7, 4.8), 4.16 (2H, m, H9, H9), 3.94 (1H, br s, OH), 3.83 (3H, s, OMe), 3.74-3.54 (4H, complex m, $\left.2 \times \mathrm{CH}_{2}-\mathrm{O}\right), 3.48$ (1H, a-t, H5, J 9.7), 2.25 (1H, a-td, $\left.\mathrm{H} 3_{\text {ax }}, J 12.5,1.5\right), 2.05$ (1H, dd, H3 eq, $J$ 12.5, 4.8 Hz), 1.85-1.40 (12H, complex m, $\left.6 \times \mathrm{CH}_{2}\right) ;{ }^{13} \mathrm{C} \mathrm{NMR}\left(75 \mathrm{MHz}, \mathrm{CDCl}_{3}\right) \delta 170.0(\mathrm{C}), 133.1$ (CH), $126.9(\mathrm{CH}), 97.3(\mathrm{C}), 97.1(\mathrm{C}), 95.3(\mathrm{C}), 72.0(\mathrm{CH}), 71.1(\mathrm{CH}), 64.3(\mathrm{CH}), 63.1\left(\mathrm{CH}_{2}\right), 60.8(3)$ $\left(\mathrm{CH}_{2}\right)$, 60.7(9) $\left(\mathrm{CH}_{2}\right), 53.5\left(\mathrm{CH}_{3}\right), 35.4\left(\mathrm{CH}_{2}\right), 28.6\left(\mathrm{CH}_{2}\right.$, two signals superimposed), $25.0(3)\left(\mathrm{CH}_{2}\right)$, 24.9(8) $\left(\mathrm{CH}_{2}\right), 18.2\left(\mathrm{CH}_{2}\right), 18.1\left(\mathrm{CH}_{2}\right)$; IR (KBr) 3468, 2948, 2873, 1751, 1439, 1272, 1209, 1191, 1148, 1071, 1045, 990, 966, $731 \mathrm{~cm}^{-1}$; MS (EI) $m / z 414\left(\mathrm{M}^{+\bullet}, 0.5 \%\right), 356(0.5), 315(0.5), 283$ (6), 196 (14), 183 (11), 168 (22), 167 (20), 155 (14), 101 (100), 83 (25); HRMS (EI) m/z calcd for $\mathrm{C}_{20} \mathrm{H}_{30} \mathrm{O}_{9}$ $\mathrm{M}^{+\bullet}:$ 414.1890. Found: 414.1890. 


\section{Compounds 15 and 16}

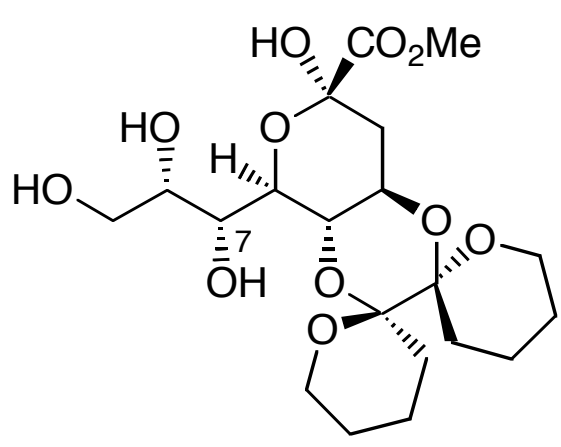

15

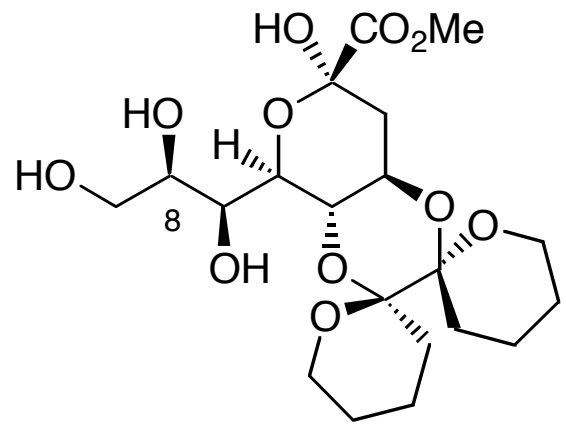

16

A magnetically stirred solution of alkene $14(50 \mathrm{mg}, 0.12 \mathrm{mmol})$ in acetone $(3 \mathrm{~mL})$ was treated with $\mathrm{H}_{2} \mathrm{O}(0.3 \mathrm{~mL})$ and $\mathrm{NMO}(28 \mathrm{mg}, 0.24 \mathrm{mmol})$ then $\mathrm{OsO}_{4}(4$ drops of a $2.5 \mathrm{wt} \%$ solution in $t$ - $\mathrm{BuOH})$. The resulting mixture was stirred at ca. $18{ }^{\circ} \mathrm{C}$ under a nitrogen atmosphere for $4.5 \mathrm{~h}$ then a solution of $\mathrm{NaHSO}_{3}(440 \mathrm{mg}, 2.3 \mathrm{mmol})$ in $\mathrm{H}_{2} \mathrm{O}(5 \mathrm{~mL})$ was added. The resulting mixture was stirred at ca. $18{ }^{\circ} \mathrm{C}$ for $1.5 \mathrm{~h}$ then filtered through a pad of Florisil. The Florisil was washed with $\mathrm{CHCl}_{3} / \mathrm{i}-\mathrm{PrOH}(100 \mathrm{~mL}$ of a 3:1 v/v mixture) and the combined filtrates concentrated under reduced pressure to give a colorless oil. Subjection of this material to column chromatography $\left(190: 9: 1 \mathrm{v} / \mathrm{v} / \mathrm{v} \mathrm{CHCl}_{3} / \mathrm{CH}_{3} \mathrm{OH} / \mathrm{AcOH}\right.$ elution) gave two fractions, $\mathrm{A}\left(\mathrm{R}_{f} 0.4\right)$ and $\mathrm{B}\left(\mathrm{R}_{f} 0.3\right)$.

Concentration of fraction A gave alcohol $15(17 \mathrm{mg}, 31 \%)$ as a colorless oil, $[\alpha]_{\mathrm{D}}^{19}+100.0(c 0.53$, $\left.\mathrm{CH}_{3} \mathrm{OH}\right) ;{ }^{1} \mathrm{H} \mathrm{NMR}\left(500 \mathrm{MHz}, \mathrm{CD}_{3} \mathrm{OD}\right) \delta 4.21$ (1H, ddd, H4, J 12.2, 9.8, 4.9), 4.03 (1H, dd, H6, J 10.3, 3.4), 3.97 (1H, m), 3.88 (1H, a-t, J 3.9), 3.86-3.58 (7H, complex m), 3.77 (3H, s, OMe), 2.05 (1H, dd, $\left.\mathrm{H} 3_{\text {eq }} J 12.2,4.9\right), 1.89\left(1 \mathrm{H}, \mathrm{a}-\mathrm{t}, \mathrm{H} 3_{\text {ax }}, J 12.2 \mathrm{~Hz}\right), 1.82-1.63\left(4 \mathrm{H}\right.$, complex m, $\left.2 \times \mathrm{CH}_{2}\right), 1.62-1.46(8 \mathrm{H}$, complex m, $4 \times \mathrm{CH}_{2}$ ); ${ }^{13} \mathrm{C}$ NMR (125.7 MHz, $\left.\mathrm{CD}_{3} \mathrm{OD}\right) \delta 171.7$ (C), 98.7 (C), 98.5 (C), 96.8 (C), 73.6 $(\mathrm{CH}), 72.8(\mathrm{CH}), 72.6(\mathrm{CH}), 70.3(\mathrm{CH}), 65.9(\mathrm{CH}), 63.8\left(\mathrm{CH}_{2}\right), 62.1\left(\mathrm{CH}_{2}\right), 61.7\left(\mathrm{CH}_{2}\right), 53.3\left(\mathrm{CH}_{3}\right)$, $37.2\left(\mathrm{CH}_{2}\right), 29.6\left(\mathrm{CH}_{2}\right), 29.4\left(\mathrm{CH}_{2}\right), 26.0\left(\mathrm{CH}_{2}\right), 25.8\left(\mathrm{CH}_{2}\right), 19.4\left(\mathrm{CH}_{2}\right), 19.1\left(\mathrm{CH}_{2}\right)$; IR $(\mathrm{NaCl}) 3369$, 2945, 1744, 1437, 1272, 1209, 1191, 1153, 1071, 1047, $988 \mathrm{~cm}^{-1}$; MS (FAB+) m/z $471\left(\mathrm{M}+\mathrm{Na}^{+}\right.$, $100 \%$ ); HRMS (FAB) $m / z$ calcd for $\mathrm{C}_{20} \mathrm{H}_{32} \mathrm{O}_{11} \mathrm{Na},\left(\mathrm{M}+\mathrm{Na}^{+}\right)$: 471.1842. Found: 471.1837.

Concentration of fraction B gave alcohol $16(20 \mathrm{mg}, 37 \%)$ as a colorless oil; $[\alpha]_{\mathrm{D}}^{19}+88.2(c 0.44$, $\left.\mathrm{CH}_{3} \mathrm{OH}\right) ;{ }^{1} \mathrm{H}$ NMR (500 MHz, CD $\left.\mathrm{OD}\right) \delta 4.18$ (1H, ddd, H4, J 12.2, 9.8, 4.9), 4.00 (1H, dd, H6, J 9.8, 1.5), 3.94 (1H, dd, $J$ 11.2, 2.9), 3.91 (1H, dd, J 5.9, 1.5), 3.84 (1H, a-t, H5, J 9.8), 3.80-3.57 (6H, complex m), 3.78 (3H, s, OMe), 2.08 (1H, dd, H3 eq $\left._{1} J 12.2,4.9\right), 1.89$ (1H, a-t, H3 ${ }_{\text {ax }}, J 12.2 \mathrm{~Hz}$ ), 1.82-1.66 (4H, complex m, $\left.2 \times \mathrm{CH}_{2}\right), 1.61-1.45\left(8 \mathrm{H}\right.$, complex m, $\left.4 \times \mathrm{CH}_{2}\right) ;{ }^{13} \mathrm{C} \mathrm{NMR}(125.7 \mathrm{MHz}$, $\left.\mathrm{CD}_{3} \mathrm{OD}\right) \delta 171.8(\mathrm{C}), 98.6(\mathrm{C}), 98.5(\mathrm{C}), 97.0(\mathrm{C}), 74.9(\mathrm{CH}), 73.5(\mathrm{CH}), 69.2(\mathrm{CH}), 68.0(\mathrm{CH}), 65.9$ 
$(\mathrm{CH}), 63.8\left(\mathrm{CH}_{2}\right), 61.8\left(\mathrm{CH}_{2}\right), 61.7\left(\mathrm{CH}_{2}\right), 53.3\left(\mathrm{CH}_{3}\right), 37.2\left(\mathrm{CH}_{2}\right), 29.6\left(\mathrm{CH}_{2}\right.$, two signals superimposed), 26.9(9) $\left(\mathrm{CH}_{2}\right), 25.9(6)\left(\mathrm{CH}_{2}\right), 19.3\left(\mathrm{CH}_{2}\right), 19.2\left(\mathrm{CH}_{2}\right)$; IR (NaCl) 3368, 2945, 1735 , 1436, 1270, 1210, 1191, 1155, 1071, 1046, $989 \mathrm{~cm}^{-1}$; MS (FAB+) m/z $471\left(\mathrm{M}+\mathrm{Na}^{+}, 100 \%\right), 413(10)$, 391 (25), 329 (40), 307 (92), 289 (48). HRMS (FAB) $m / z$ calcd for $\mathrm{C}_{20} \mathrm{H}_{32} \mathrm{O}_{11} \mathrm{Na}\left(\mathrm{M}+\mathrm{Na}^{+}\right)$: 471.1842. Found: 471.1845.

\section{Compound 7-epi-3}

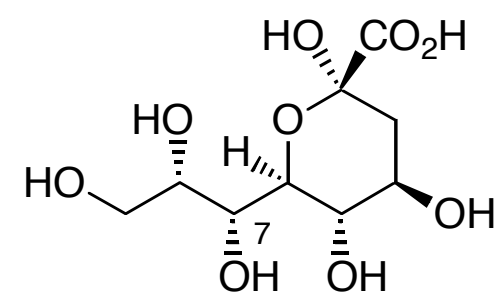

7-epi-3

Compound $15(11 \mathrm{mg}, 0.025 \mathrm{mmol})$ was dissolved in $\mathrm{TFA} / \mathrm{H}_{2} \mathrm{O}(1.0 \mathrm{~mL}$ of a 95:5 v/v mixture $)$ and the resulting solution was stirred at $18{ }^{\circ} \mathrm{C}$ for $24 \mathrm{~h}$. ESMS (+ve and -ve ionization) analysis after this time suggested some starting material remained so the reaction mixture was concentrated under reduced pressure and the residue thus obtained was again treated with $\mathrm{TFA} / \mathrm{H}_{2} \mathrm{O}(1.0 \mathrm{~mL}$ of a 95:5 v/v mixture) at $18{ }^{\circ} \mathrm{C}$ for $16 \mathrm{~h}$. The ensuing mixture was then concentrated under reduced pressure with toluene coevaporation. The residue thus obtained was dissolved in $\mathrm{H}_{2} \mathrm{O}(1.5 \mathrm{~mL})$, treated with $\mathrm{NaOH}(50 \mu \mathrm{L}$ of a $1 \mathrm{M}$ aq solution), stirred at $18{ }^{\circ} \mathrm{C}$ for $2 \mathrm{~h}$ then diluted with $\mathrm{H}_{2} \mathrm{O}(1 \mathrm{~mL})$. Amberlite IR $120\left(\mathrm{H}^{+}\right)$ion exchange resin was then added and after stirring for $0.25 \mathrm{~h}$ the reaction mixture was filtered $\left(\mathrm{H}_{2} \mathrm{O}\right.$ washes, $2 \mathrm{~mL}$ ) and the filtrate concentrated under reduced pressure. The residue was dissolved in $\mathrm{H}_{2} \mathrm{O}$ $(0.5 \mathrm{~mL})$ and applied to a $\mathrm{C} 18$ reverse-phase solid-phase extraction column $\left(\mathrm{H}_{2} \mathrm{O}\right.$ elution). Concentration of the appropriate fractions yielded sialic acid 7-epi-3 (5 mg, 76\%) as a light-yellow gum, ${ }^{1} \mathrm{H}$ NMR (500 MHz, $\left.\mathrm{D}_{2} \mathrm{O}\right) \delta$ 4.00-3.94 (2H, complex m, H4, H8), 3.92 (1H, dd, H7, J 3.4, 2.9), 3.86 (1H, dd, H6, J 9.8, 3.4), 3.69 (1H, dd, H9, J 11.5, 4.9), 3.65 (1H, dd, H9', J 11.5, 7.1), 3.58 (1H, a-t, H5, J 9.5), $3.29(1 \mathrm{H}, \mathrm{m}, \mathrm{OH}), 2.19\left(1 \mathrm{H}, \mathrm{dd}, \mathrm{H} 3_{\mathrm{eq}}, J 12.5,5.0\right), 1.81\left(1 \mathrm{H}, \mathrm{a}-\mathrm{t}, \mathrm{H} 3_{\mathrm{ax}}, J 12.5 \mathrm{~Hz}\right)$; ${ }^{13} \mathrm{C}$ NMR $\left(150 \mathrm{MHz}, \mathrm{D}_{2} \mathrm{O} / \mathrm{CD}_{3} \mathrm{CN}\right) \delta 74.9,72.7,72.4,71.7,69.8,63.7,39.7$ (signals due to $\mathrm{C} 1$ and $\mathrm{C} 2$ not observed). 


\section{Compound 17}

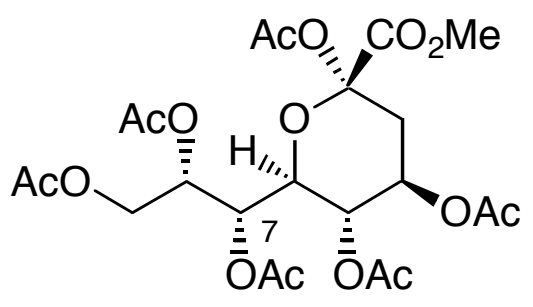

17

Compound $15(9 \mathrm{mg}, 0.020 \mathrm{mmol})$ was dissolved in $\mathrm{TFA} / \mathrm{H}_{2} \mathrm{O}(1.0 \mathrm{~mL}$ of a $95: 5 \mathrm{v} / \mathrm{v}$ mixture $)$ and the resulting solution was stirred at $18{ }^{\circ} \mathrm{C}$ for $24 \mathrm{~h}$. ESMS (+ve and -ve ionization) analysis after this time suggested some starting material remained so the reaction mixture was concentrated under reduced pressure and the residue thus obtained was again treated with $\mathrm{TFA} / \mathrm{H}_{2} \mathrm{O}(1.0 \mathrm{~mL}$ of a 95:5 v/v mixture $)$ at $18{ }^{\circ} \mathrm{C}$ for $16 \mathrm{~h}$. The ensuing mixture was then concentrated under reduced pressure with toluene coevaporation to yield sialic acid 7-epi-3 which was used directly in the next step.

Thus, crude acid 7-epi-3, obtained as described immediately above, was dissolved in $\mathrm{CH}_{3} \mathrm{OH}(1 \mathrm{~mL})$ and the resulting solution treated with AG 50W-X8 (200-400 mesh, $\mathrm{H}^{+}$form, $10 \mathrm{mg}$ ) cation exchange resin. After stirring at $18{ }^{\circ} \mathrm{C}$ for $16 \mathrm{~h}$, TLC analysis $\left(60: 30: 3: 5 \mathrm{v} / \mathrm{v} / \mathrm{v} / \mathrm{v} \mathrm{CHCl}_{3} / \mathrm{CH}_{3} \mathrm{OH} / \mathrm{AcOH}_{2} \mathrm{H}_{2} \mathrm{O}\right.$ elution) showed the formation of a new product $\left(\mathrm{R}_{f} 0.4\right)$. Consequently, the reaction mixture was filtered through a sintered funnel with $\mathrm{CH}_{3} \mathrm{OH}$ washes $(20 \mathrm{~mL})$ and the combined filtrates concentrated under reduced pressure, with toluene co-evaporation, to give a light-yellow oil. This was treated directly with pyridine $(0.5 \mathrm{~mL})$, acetic anhydride $(0.5 \mathrm{~mL})$ and DMAP $(4 \mathrm{mg})$ then the resulting solution was stirred at $18{ }^{\circ} \mathrm{C}$ for $18 \mathrm{~h}$. TLC analysis $(1: 1 \mathrm{v} / \mathrm{v}$ EtOAc/hexane elution) after this time showed the formation of a new product $\left(\mathrm{R}_{f} 0.3\right)$. Concentration of the reaction mixture under reduced pressure, with toluene co-evaporation, gave a residue that was subjected to flash chromatography (1:2 $\mathrm{v} / \mathrm{v} \mathrm{EtOAc} / \mathrm{hexane}$ elution). Concentration of the appropriate fractions $\left(\mathrm{R}_{f} 0.3,1: 1 \mathrm{v} / \mathrm{v}\right.$ EtOAc/hexane) then gave compound 17 (6 mg, 56\% over 3 steps) as a colorless glass, mp $43-45{ }^{\circ} \mathrm{C} ;[\alpha]_{\mathrm{D}}^{18}+28.7(c$ 0.23, $\left.\mathrm{CHCl}_{3}\right) ;{ }^{1} \mathrm{H} \mathrm{NMR}\left(500 \mathrm{MHz}, \mathrm{CDCl}_{3}\right) \delta 5.44(1 \mathrm{H}, \mathrm{a}-\mathrm{dt}, \mathrm{H} 8, J$ 6.3, 4.4, ), 5.25 (1H, ddd, H4, J 11.2, 9.3, 4.9), 5.20 (1H, a-t, H5, J 9.3), 5.17 (1H, a-t, H7, J 3.9), 4.23 (1H, dd, H9, J 12.2, 4.4), 4.18 (1H, dd, H9', J 12.2, 6.3), 4.09 (1H, dd, H6, J 9.8, 3.9), 3.78 (3H, s, OMe), 2.55 (1H, dd, H3, J 13.7, 4.9 Hz), $2.14\left(3 \mathrm{H}, \mathrm{s}, \mathrm{CH}_{3} \mathrm{CO}\right), 2.10\left(3 \mathrm{H}, \mathrm{s}, \mathrm{CH}_{3} \mathrm{CO}\right), 2.08(2)\left(3 \mathrm{H}, \mathrm{s}, \mathrm{CH}_{3} \mathrm{CO}\right), 2.07(7)\left(3 \mathrm{H}, \mathrm{s}, \mathrm{CH}_{3} \mathrm{CO}\right), 2.05(3 \mathrm{H}$, s, $\left.\mathrm{CH}_{3} \mathrm{CO}\right), 2.03\left(1 \mathrm{H}, \mathrm{m}, \mathrm{H} 3\right.$ ', obscured), $2.02\left(3 \mathrm{H}, \mathrm{s}, \mathrm{CH}_{3} \mathrm{CO}\right) ;{ }^{13} \mathrm{C} \mathrm{NMR}\left(125.7 \mathrm{MHz}, \mathrm{CDCl}_{3}\right) \delta 170.5$ 
(C), 170.0(9) (C), 170.0(5) (C), 169.7 (C), 169.6 (C), 168.1 (C), 166.2 (C), 96.7 (C), 72.4 (CH), 69.6 $(\mathrm{CH}), 69.2(\mathrm{CH}), 68.7(\mathrm{CH}), 68.6(\mathrm{CH}), 62.4\left(\mathrm{CH}_{2}\right), 53.2\left(\mathrm{CH}_{3}\right), 35.3\left(\mathrm{CH}_{2}\right), 20.8(5)\left(\mathrm{CH}_{3}\right), 20.8(3)$ $\left(\mathrm{CH}_{3}\right), 20.6(8)\left(\mathrm{CH}_{3}\right), 20.6(5)\left(\mathrm{CH}_{3}\right)$ (two signals obscured or overlapping); IR ( $\mathrm{NaCl}$, thin film) 2958, 1747, 1436, 1371, 1222, 1048, 1012, $939 \mathrm{~cm}^{-1}$; MS (ESI+) 557 (M+Na+, 100); HRMS (FAB) $m / z$ calcd for $\mathrm{C}_{22} \mathrm{H}_{30} \mathrm{O}_{15} \mathrm{Cs}\left(\mathrm{M}+\mathrm{Cs}^{+}\right)$: 667.0634. Found: 667.0647 .

\section{Compound 8-epi-3}

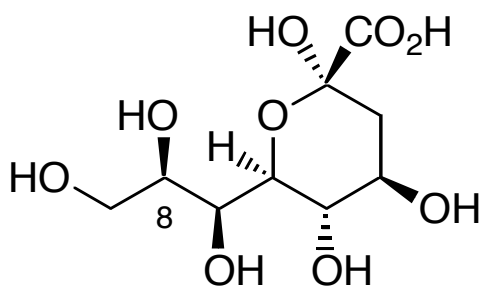

8-epi-3

Compound 16 (9 mg, $0.020 \mathrm{mmol})$ was dissolved in $\mathrm{TFA} / \mathrm{H}_{2} \mathrm{O}(1.0 \mathrm{~mL}$ of a 95:5 v/v mixture) and the resulting solution was stirred at $18{ }^{\circ} \mathrm{C}$ for $24 \mathrm{~h}$. ESMS (+ve and -ve ionization) analysis after this time suggested some starting material remained so the reaction mixture was concentrated under reduced pressure and the residue thus obtained was again treated with $\mathrm{TFA} / \mathrm{H}_{2} \mathrm{O}(1.0 \mathrm{~mL}$ of a 95:5 v/v mixture) at $18{ }^{\circ} \mathrm{C}$ for $16 \mathrm{~h}$. Concentration of the ensuing mixture under reduced pressure, with toluene coevaporation, yielded a light-yellow oil. This was dissolved in $\mathrm{H}_{2} \mathrm{O}(1.5 \mathrm{~mL})$, treated with $\mathrm{NaOH}(50 \mu \mathrm{L}$ of a $1 \mathrm{M}$ aq solution), stirred at $18^{\circ} \mathrm{C}$ for $2 \mathrm{~h}$ then diluted with $\mathrm{H}_{2} \mathrm{O}(1 \mathrm{~mL})$. Amberlite IR $120\left(\mathrm{H}^{+}\right)$ion exchange resin then was added and, after stirring for $0.25 \mathrm{~h}$, the reaction mixture was filtered $\left(\mathrm{H}_{2} \mathrm{O}\right.$ washes, $2 \mathrm{~mL}$ ) and the combined filtrates concentrated under reduced pressure. The residue thus obtained was dissolved in $\mathrm{H}_{2} \mathrm{O}(0.5 \mathrm{~mL})$ and applied to a $\mathrm{C} 18$ reverse-phase solid-phase extraction column $\left(\mathrm{H}_{2} \mathrm{O}\right.$ elution). Concentration of the appropriate fractions yielded sialic acid 8-epi-3 (4 mg, $69 \%$ ) as a light-yellow gum, ${ }^{1} \mathrm{H}$ NMR (500 MHz, $\left.\mathrm{D}_{2} \mathrm{O}\right) \delta$ 3.96-3.89 (2H, complex m, H7, H4), 3.82 (1H, m, H8), 3.75 (1H, d, H6, J 9.5), 3.69 (1H, dd, H9, J 12.0, 3.0), 3.58 (1H, a-t, H5, J 9.5), 3.56 (1H, dd H9, $J$ 12.0, 6.3), 2.18 (1H, dd, H3 $\left.{ }_{\text {eq }}, J 12.5,4.9\right), 1.77\left(1 \mathrm{H}, \mathrm{a}-\mathrm{t}, \mathrm{H} 3_{\mathrm{ax}}, J 12.5 \mathrm{~Hz}\right) ;{ }^{13} \mathrm{C} \mathrm{NMR}(150$ $\left.\mathrm{MHz}, \mathrm{D}_{2} \mathrm{O} / \mathrm{CD}_{3} \mathrm{CN}\right) \delta 74.2,74.1,71.4,69.8,69.5,63.1,40.0$ (signals due to $\mathrm{C} 1$ and $\mathrm{C} 2$ not observed). 


\section{Compound 18}

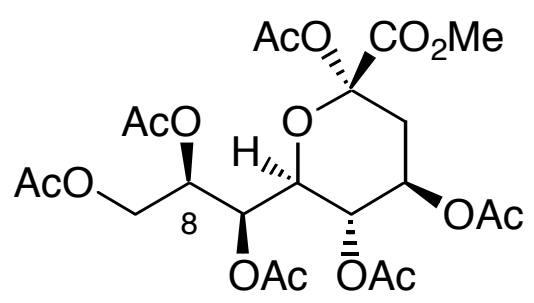

18

Compound 16 (7 mg, $0.016 \mathrm{mmol})$ was dissolved in TFA/ $\mathrm{H}_{2} \mathrm{O}(0.5 \mathrm{~mL}$ of a 95:5 v/v mixture) and the resulting solution was stirred at $18{ }^{\circ} \mathrm{C}$ for $24 \mathrm{~h}$. TLC analysis $\left(10 \% \mathrm{CH}_{3} \mathrm{OH} / \mathrm{CHCl}_{3}\right)$ after this time showed an absence of starting material $\left(\mathrm{R}_{f} 0.25\right)$ and the formation of a new product $\left(\mathrm{R}_{f} 0.0\right)$. Concentration of the reaction mixture under reduced pressure with toluene co-evaporation yielded 8epi-3 as a light-yellow gum. This gum was dissolved in $\mathrm{CH}_{3} \mathrm{OH}(1 \mathrm{~mL})$ and the resulting solution treated with AG 50W-X8 (200-400 mesh, $\mathrm{H}^{+}$form, $10 \mathrm{mg}$ ) cation exchange resin at $18{ }^{\circ} \mathrm{C}$ for $16 \mathrm{~h}$. TLC analysis (60:30:3:5 v/v/v/v $\mathrm{CHCl}_{3} / \mathrm{CH}_{3} \mathrm{OH} / \mathrm{AcOH} / \mathrm{H}_{2} \mathrm{O}$ elution) after this time showed the formation of a new product $\left(\mathrm{R}_{f} 0.3\right)$. Filtration through a sintered funnel with $\mathrm{CH}_{3} \mathrm{OH}$ washes and concentration of the filtrates under reduced pressure, with toluene co-evaporation, gave a yellow oil which was treated with pyridine $(0.5 \mathrm{~mL})$, acetic anhydride $(0.5 \mathrm{~mL})$ and DMAP $(4 \mathrm{mg})$. The resulting solution was stirred at $18{ }^{\circ} \mathrm{C}$ for $18 \mathrm{~h}$, after which time TLC analysis $(1: 1 \mathrm{v} / \mathrm{v} \mathrm{EtOAc/hexane} \mathrm{elution)}$ showed the formation of a new product $\left(\mathrm{R}_{f} 0.3\right)$. The reaction mixture was concentrated under reduced pressure, again with toluene co-evaporation. The residue thus obtained was subjected to flash chromatography $\left(1: 1 \mathrm{v} / \mathrm{v}\right.$ EtOAc/hexane elution) and concentration of the appropriate fractions $\left(\mathrm{R}_{f} 0.3\right.$, 1:1 v/v EtOAc/hexane) gave compound 18 (4 mg, 50\% over 3 steps) as a colorless solid, $\mathrm{mp}$ 136-138 ${ }^{\circ} \mathrm{C} ;[\alpha]_{\mathrm{D}}^{18}+12.0\left(c 0.15, \mathrm{CHCl}_{3}\right) ;{ }^{1} \mathrm{H} \mathrm{NMR}\left(500 \mathrm{MHz}, \mathrm{CDCl}_{3}\right) \delta$ 5.36-5.26 (3H, complex m, H4, H7, H8), 5.03 (1H, a-t, H5, J 10.3), 4.33 (1H, dd, H9, J 13.2, 2.4), 4.26 (1H, dd, H9', J 13.2, 4.4), 4.12 (1H, dd, H6, J 10.3, 2.4), 3.81 (3H, s, OMe), 2.62 (1H, dd, H3, J 13.7, 5.4 Hz), 2.17 (3H, s, $\mathrm{CH}_{3} \mathrm{CO}$ ), 2.10 (3H, s, $\left.\mathrm{CH}_{3} \mathrm{CO}\right), 2.09$ (3H, s, $\left.\mathrm{CH}_{3} \mathrm{CO}\right), 2.08$ (1H, m, H3', obscured), 2.04 (3H, s, $\left.\mathrm{CH}_{3} \mathrm{CO}\right), 2.02(1)\left(3 \mathrm{H}, \mathrm{s}, \mathrm{CH}_{3} \mathrm{CO}\right), 2.01(5)\left(3 \mathrm{H}, \mathrm{s}, \mathrm{CH}_{3} \mathrm{CO}\right) ;{ }^{13} \mathrm{C} \mathrm{NMR}\left(125.7 \mathrm{MHz}, \mathrm{CDCl}_{3}\right) \delta 170.6(\mathrm{C})$, 170.2 (C), $170.1(\mathrm{C}), 169.7(3)(\mathrm{C}), 169.6(9)(\mathrm{C}), 168.7$ (C), 166.1 (C), 97.2 (C), $71.2(\mathrm{CH}), 70.8(\mathrm{CH})$, $68.4(\mathrm{CH}), 67.5(\mathrm{CH}), 67.0(\mathrm{CH}), 62.4\left(\mathrm{CH}_{2}\right), 53.3\left(\mathrm{CH}_{3}\right), 35.6\left(\mathrm{CH}_{2}\right), 20.8\left(\mathrm{CH}_{3}\right), 20.7\left(\mathrm{CH}_{3}\right), 20.6$ $\left(\mathrm{CH}_{3}\right)$ (three signals due to acetate methyl groups obscured or overlapping); IR ( $\mathrm{NaCl}$, thin film) 2924, 2854, 1748, 1441, 1371, 1228, 1112, 1055, $1011 \mathrm{~cm}^{-1}$; MS (ESI+) 557 (M+Na+, 100\%), 497 (10). HRMS (FAB) $m / z$ calcd for $\mathrm{C}_{22} \mathrm{H}_{30} \mathrm{O}_{15} \mathrm{Cs}\left(\mathrm{M}+\mathrm{Cs}^{+}\right)$: 667.0634. Found: 667.0612. 


\section{Compound 19}

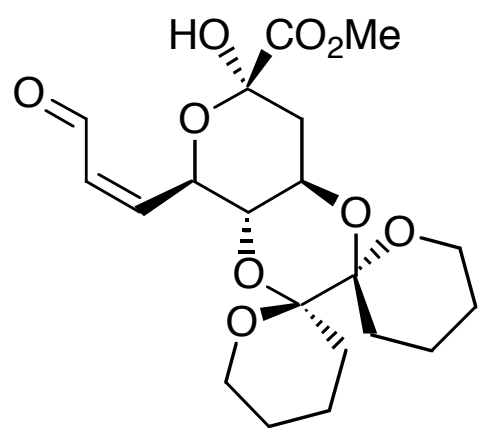

19

Compound 13 (69 mg, $0.168 \mathrm{mmol}$ ) was dissolved in dry, degassed benzene and the resulting solution irradiated at $300 \mathrm{~nm}$ (Rayonet reactor) for $2 \mathrm{~h}$. TLC analysis (1:1 v/v EtOAc/hexane elution) after this time showed a mixture of starting material $\left(\mathrm{R}_{f} 0.3\right)$ and product $\left(\mathrm{R}_{f} 0.2\right)$, while ${ }^{1} \mathrm{H}$ NMR analysis showed a 1.2:1 ratio of compounds $\mathbf{1 3}$ and 19. The reaction mixture was concentrated under reduced pressure and the residue thus obtained subjected to flash chromatography $(1: 3 \mathrm{v} / \mathrm{v}$ EtOAc/hexane with $0.1 \% \mathrm{Et}_{3} \mathrm{~N}$ elution) and thus affording two fractions, $\mathrm{A}\left(\mathrm{R}_{f} 0.2\right)$ and $\mathrm{B}\left(\mathrm{R}_{f} 0.3\right)$.

Concentration of fraction A gave compound $19(29 \mathrm{mg}, 42 \%)$ as a colorless foam, $[\alpha]_{\mathrm{D}}+97.1(c 0.93$, $\left.\mathrm{CH}_{2} \mathrm{Cl}_{2}\right) ;{ }^{1} \mathrm{H} \mathrm{NMR}\left(300 \mathrm{MHz}, \mathrm{C}_{6} \mathrm{D}_{6}\right) \delta 9.99(1 \mathrm{H}, \mathrm{d}, \mathrm{H} 9, J$ 8.1), 6.17 (1H, dd, H7, J 11.5, 7.4), 5.89 (1H, ddd, H8, J 11.5, 8.1 , 1.4), 5.19 (1H, ddd, H6, J 10.2, 7.4, 1.4), 4.56 (1H, ddd, H4, J 12.2, 9.3, 4.8), 4.02 (1H, m, OH), 3.62 (1H, a-t, H5, J 9.6), 3.65-3.35 (3H, complex m), 3.25 (1H, a-d, J 9.3), 3.20 (3H, s, OMe), 2.41 (1H, a-t, H3 $3_{\text {ax }} J$ 12.4), 2.15 (1H, dd, H3 ${ }_{\text {eq }} J$ 12.4, 4.8 Hz), 2.16-1.95 (2H, complex m), 1.88-1.05 (10H, complex m); ${ }^{13} \mathrm{C}$ NMR (75 MHz, $\left.\mathrm{C}_{6} \mathrm{D}_{6}\right) \delta 191.0(\mathrm{CH}), 169.9(\mathrm{C}), 144.6(\mathrm{CH}), 132.4$ $(\mathrm{CH}), 98.3(\mathrm{C}), 97.6(\mathrm{C}), 95.8(\mathrm{C}), 70.1(\mathrm{CH}), 69.1(\mathrm{CH}), 64.7(\mathrm{CH}), 60.8\left(\mathrm{CH}_{2}\right), 60.6\left(\mathrm{CH}_{2}\right), 52.8$ $\left(\mathrm{CH}_{3}\right), 35.8\left(\mathrm{CH}_{2}\right), 29.0\left(\mathrm{CH}_{2}\right), 28.8\left(\mathrm{CH}_{2}\right), 25.2\left(\mathrm{CH}_{2}\right), 25.0\left(\mathrm{CH}_{2}\right), 18.6\left(\mathrm{CH}_{2}\right), 18.4\left(\mathrm{CH}_{2}\right) ; \mathrm{IR}(\mathrm{NaCl})$ 3436, 2927, 2873, 1752, 1686, 1439, 1272, 1210, 1191, 1147, 1086, 1070, 1047, 990, 965, 936, 898, $878 \mathrm{~cm}^{-1}$; MS (ESI+) $\mathrm{m} / z 451\left(\mathrm{M}+\mathrm{K}^{+}, 15 \%\right), 435\left(\mathrm{M}+\mathrm{Na}^{+}, 100\right)$; HRMS (FAB) $\mathrm{m} / z$ calcd for $\mathrm{C}_{20} \mathrm{H}_{28} \mathrm{O}_{9} \mathrm{Cs}\left(\mathrm{M}+\mathrm{Cs}^{+}\right):$545.0788. Found: 545.0775.

Concentration of fraction B gave starting material 13 (40 mg, 58\% recovery) as a colorless foam and identical, in all respects, with authentic material. 


\section{Luche reduction of Compound 19 - Formation of the Corresponding Allylic Alcohol}

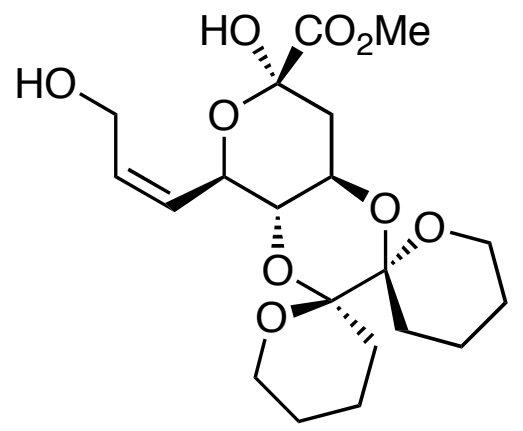

$\mathrm{CeCl}_{3} \cdot 7 \mathrm{H}_{2} \mathrm{O}(59 \mathrm{mg}, 0.158 \mathrm{mmol})$ was added to a magnetically stirred solution of aldehyde 19 (59 $\mathrm{mg}$, $0.143 \mathrm{mmol})$ and 2,6-lutidine $(77 \mathrm{mg}, 83 \mu \mathrm{L}, 0.716 \mathrm{mmol})$ in $\mathrm{CH}_{2} \mathrm{Cl}_{2} / \mathrm{EtOH}(2.6 \mathrm{~mL}$ of a $1: 1 \mathrm{mixture})$ maintained at ca. $18^{\circ} \mathrm{C}$. This mixture was then cooled to $-78^{\circ} \mathrm{C}$ and treated with $\mathrm{NaBH}_{4}(16 \mathrm{mg}, 0.430$ $\mathrm{mmol})$ in $\mathrm{EtOH}(0.43 \mathrm{~mL})$. Stirring was continued at $-78^{\circ} \mathrm{C}$ for $1 \mathrm{~h}$ at which time TLC analysis $(1: 2$ $\mathrm{v} / \mathrm{v}$ EtOAc/hexane elution) showed an absence of starting material $\left(\mathrm{R}_{f} 0.3\right)$ and the presence of a product $\left(\mathrm{R}_{f} 0.1\right)$. Consequently acetone $(0.5 \mathrm{~mL})$ was added to the reaction mixture, the cooling bath was then removed and the reaction mixture poured into $\mathrm{H}_{2} \mathrm{O}(40 \mathrm{~mL}) . \mathrm{HCl}(5 \mathrm{~mL}$ of a $1 \mathrm{M}$ aq solution) was added and the resulting mixture extracted with $\mathrm{CH}_{2} \mathrm{Cl}_{2}(3 \times 20 \mathrm{~mL})$. The combined organic phases were washed with brine $(1 \times 15 \mathrm{~mL})$ then dried $\left(\mathrm{Na}_{2} \mathrm{SO}_{4}\right)$, filtered and concentrated under reduced pressure to give a yellow oil. Subjection of this material to flash chromatography $(1: 1 \mathrm{v} / \mathrm{v}$ EtOAc/hexane elution) and concentration of the appropriate fractions $\left(\mathrm{R}_{f} 0.2\right)$ under reduced pressure gave the title allylic alcohol $(23 \mathrm{mg}, 39 \%)$ as a clear, colorless oil, $[\alpha]_{\mathrm{D}}^{18}+91.7\left(c 0.90, \mathrm{CHCl}_{3}\right) ;{ }^{1} \mathrm{H}$ NMR (300 MHz, $\left.\mathrm{CDCl}_{3}\right) \delta 5.99$ (1H, m, H8), 5.54 (1H, m, H7), 4.88 (1H, ddd, H6, J 9.9, 8.3, 1.1), 4.30-4.08 (3H, complex m), 3.82 (3H, s, OMe), 3.80-3.55 (4H, complex m), 3.48 (1H, a-t, H5, J 9.8), $2.56\left(1 \mathrm{H}\right.$, br s, OH), 2.24 (1H, a-t, H3 $\left.3_{\text {ax }}, J 12.5\right), 2.06\left(1 \mathrm{H}, \mathrm{dd}, \mathrm{H} 3_{\text {eq }}, J 12.5,4.8 \mathrm{~Hz}\right), 1.82-1.42(12 \mathrm{H}$, complex m, $\left.6 \times \mathrm{CH}_{2}\right) ;{ }^{13} \mathrm{C}$ NMR $\left(75 \mathrm{MHz}, \mathrm{CDCl}_{3}\right) \delta 170.0(\mathrm{C}), 133.7(\mathrm{CH}), 128.6(\mathrm{CH}), 97.8(\mathrm{C}), 97.1$ (C), $95.4(\mathrm{C}), 70.3(\mathrm{CH}), 68.4(\mathrm{CH}), 64.2(\mathrm{CH}), 60.7\left(\mathrm{CH}_{2}\right), 58.7\left(\mathrm{CH}_{2}\right), 53.4\left(\mathrm{CH}_{3}\right), 35.3\left(\mathrm{CH}_{2}\right), 28.6$ $\left(\mathrm{CH}_{2}\right), 28.2\left(\mathrm{CH}_{2}\right), 24.8\left(\mathrm{CH}_{2}\right), 24.7\left(\mathrm{CH}_{2}\right), 18.0\left(\mathrm{CH}_{2}\right)$ (two signals obscured or overlapping); IR ( $\mathrm{NaCl}$, thin film) 3468, 2947, 2874, 1751, 1439, 1272, 1209, 1191, 1151, 1071, 1046, 990, 937, 899, 877, $754 \mathrm{~cm}^{-1}$; MS (ESI+) $m / z, 437\left(\mathrm{M}+\mathrm{Na}^{+}, 100 \%\right)$; HRMS (FAB) $m / z$ calcd for $\mathrm{C}_{20} \mathrm{H}_{30} \mathrm{O}_{9} \mathrm{Cs}\left(\mathrm{M}+\mathrm{Cs}^{+}\right)$: 547.0944. Found: 547.0926. 


\section{Compound 20}

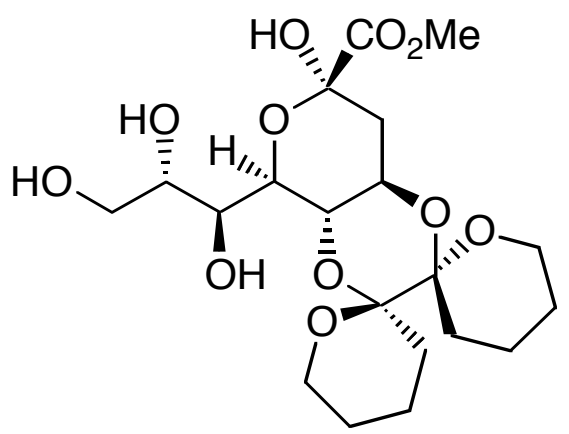

20

A magnetically stirred solution of the allylic alcohol derived from the Luche reduction of compound $\mathbf{1 9}$ (20 mg, $0.048 \mathrm{mmol})$ in acetone $(1.5 \mathrm{~mL})$ was treated with $\mathrm{H}_{2} \mathrm{O}(0.15 \mathrm{~mL})$ and NMO (11 mg, 0.096 mmol) then $\mathrm{OsO}_{4}$ ( 2 drops of a $2.5 \mathrm{wt} \%$ solution in $\left.t-\mathrm{BuOH}\right)$ was added and the ensuing mixture stirred at $18{ }^{\circ} \mathrm{C}$ under a nitrogen atmosphere for $16 \mathrm{~h}$. TLC analysis $\left(10 \% \mathrm{v} / \mathrm{v} \mathrm{CH}_{3} \mathrm{OH} / \mathrm{CHCl}_{3}\right.$ elution) after this time showed residual starting material $\left(\mathrm{R}_{f} 0.6\right)$ and a major product spot $\left(\mathrm{R}_{f} 0.3\right)$. Consequently, additional NMO (11 mg, $0.096 \mathrm{mmol}$ ), $\mathrm{OsO}_{4}$ (2 drops of a $2.5 \mathrm{wt} \%$ solution in $t$ - $\mathrm{BuOH}$ ) and THF (0.5 $\mathrm{mL}$ ) were added and the reaction mixture then stirred at $18{ }^{\circ} \mathrm{C}$ for an additional $64 \mathrm{~h}$. Further $\mathrm{OsO}_{4}$ (1 drop of a $2.5 \mathrm{wt} \%$ solution in $t-\mathrm{BuOH})$ and THF $(0.5 \mathrm{~mL})$ were added after this time and stirring was continued for another $16 \mathrm{~h}$. After this time TLC analysis $\left(10 \% \mathrm{CH}_{3} \mathrm{OH} / \mathrm{CHCl}_{3}\right.$ elution) showed an absence of starting material $\left(\mathrm{R}_{f} 0.6\right)$. As a result, solid $\mathrm{Na}_{2} \mathrm{~S}_{2} \mathrm{O}_{5}(80 \mathrm{mg}), \mathrm{H}_{2} \mathrm{O}(0.2 \mathrm{~mL})$ and Florisil $(0.2$ g) were added and the resulting mixture was stirred at $18^{\circ} \mathrm{C}$ for $4 \mathrm{~h}$. Silica gel $(0.2 \mathrm{~g})$ was then added and the solvent removed under reduced pressure. Application of this material to flash chromatography (5\% v/v CH $\mathrm{CH}_{3} \mathrm{OH} / \mathrm{CHCl}_{3}$ containing $0.05 \% \mathrm{NH}_{3}$ elution) and concentration of the appropriate fraction $\left(\mathrm{R}_{f} 0.3,10 \% \mathrm{v} / \mathrm{v} \mathrm{CH}_{3} \mathrm{OH} / \mathrm{CHCl}_{3}\right)$ gave compound $20(93 \mathrm{mg}, 42 \%)$ as a clear, colorless oil, $[\alpha]_{\mathrm{D}}^{17}+74.2$ (c 0.52, $\left.\mathrm{CH}_{3} \mathrm{OH}\right) ;{ }^{1} \mathrm{H}$ NMR (500 MHz, $\left.\mathrm{CDCl}_{3}\right) \delta 4.21(1 \mathrm{H}, \mathrm{ddd}, \mathrm{H} 4, J$ 12.2, 9.8, 4.9), $4.18(1 \mathrm{H}, \mathrm{dd}, \mathrm{H} 6$, $J$ 9.8, 1.5), 3.95-3.56 (9H, complex m), 3.78 (3H, s, OMe), $2.06\left(1 \mathrm{H}, \mathrm{dd}, \mathrm{H} 3_{\text {eq }}, J 12.7,4.9\right), 1.91(1 \mathrm{H}$, a-t, $\left.\mathrm{H}_{\text {ax }}, J 12.7 \mathrm{~Hz}\right), 1.84-1.69\left(4 \mathrm{H}\right.$, complex m, $\left.2 \times \mathrm{CH}_{2}\right), 1.61-1.45\left(8 \mathrm{H}\right.$, complex m, $\left.4 \times \mathrm{CH}_{2}\right) ;{ }^{13} \mathrm{C}$ NMR (125.7 MHz, $\mathrm{CDCl}_{3}$ ) $\delta 172.1(\mathrm{C}), 98.5$ (C, two signals superimposed), $97.1(\mathrm{C}), 72.2(\mathrm{CH}), 71.5$ $(\mathrm{CH}), 69.2(\mathrm{CH}), 67.6(\mathrm{CH}), 66.2(\mathrm{CH}), 64.9\left(\mathrm{CH}_{2}\right), 61.8\left(\mathrm{CH}_{2}\right), 61.7\left(\mathrm{CH}_{2}\right), 53.3\left(\mathrm{CH}_{3}\right), 37.1\left(\mathrm{CH}_{2}\right)$, 29.6 $\left(\mathrm{CH}_{2}\right.$, two signals superimposed), $26.0\left(\mathrm{CH}_{2}\right.$, two signals superimposed $), 19.3\left(\mathrm{CH}_{2}\right), 19.2\left(\mathrm{CH}_{2}\right)$; IR ( $\mathrm{NaCl}$, thin film) 3369, 2945, 2868, 1735, 1454, 1437, 1272, 1209, 1191, 1153, 1070, 1047, 989 $\mathrm{cm}^{-1} ; \mathrm{MS}(\mathrm{ESI}+) \mathrm{m} / z$ 471(M+Na+ $\left.{ }^{+}, 100 \%\right), 413(25)$. 


\section{Compound 21}

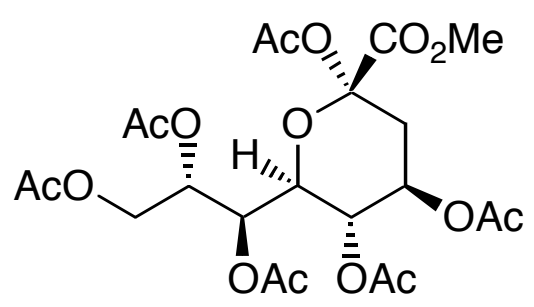

21

Compound $20(9.3 \mathrm{mg}, 0.021 \mathrm{mmol})$ was dissolved in TFA/ $\mathrm{H}_{2} \mathrm{O}(1.0 \mathrm{~mL}$ of a 95:5 v/v mixture $)$ and the resulting solution was stirred at $18^{\circ} \mathrm{C}$ for $24 \mathrm{~h}$. ESMS (+ve and -ve ionization) analysis of the reaction mixture after this time suggested some starting material remained so the reaction mixture was concentrated under reduced pressure and the residue thus obtained was again treated with TFA/ $\mathrm{H}_{2} \mathrm{O}$ $\left(1.0 \mathrm{~mL}\right.$ of a $95: 5 \mathrm{v} / \mathrm{v}$ mixture) at $18{ }^{\circ} \mathrm{C}$ for $16 \mathrm{~h}$. Concentration of the ensuing oil under reduced pressure, with toluene co-evaporation, then yielded compound $\mathbf{3}$ which was used directly in the next step.

The sample of compound $\mathbf{3}$ obtained as described immediately above was dissolved in $\mathrm{CH}_{3} \mathrm{OH}(1 \mathrm{~mL})$ and the resulting solution treated with AG 50W-X8 (200-400 mesh, $\mathrm{H}^{+}$form, $10 \mathrm{mg}$ ) cation exchange resin at $18{ }^{\circ} \mathrm{C}$ for $16 \mathrm{~h}$. TLC analysis (60:30:3:5 v/v/v/v $\mathrm{CHCl}_{3} / \mathrm{CH}_{3} \mathrm{OH} / \mathrm{AcOH} / \mathrm{H}_{2} \mathrm{O}$ elution) after this time showed the formation of a new product $\left(\mathrm{R}_{f} 0.3\right)$. Filtration of the reaction mixture through a sintered funnel with $\mathrm{CH}_{3} \mathrm{OH}$ washes and concentration of the combined filtrates under reduced pressure gave a residue which was treated directly with pyridine $(0.5 \mathrm{~mL})$, acetic anhydride $(0.5 \mathrm{~mL})$ and DMAP (4 mg). The resulting solution was stirred at $18{ }^{\circ} \mathrm{C}$ for $18 \mathrm{~h}$ at which time TLC analysis $(1: 1 \mathrm{v} / \mathrm{v}$ EtOAc/hexane elution) showed the formation of a new product $\left(\mathrm{R}_{f} 0.3\right)$. Concentration of the reaction mixture under reduced pressure, with toluene co-evaporation, gave a residue that was subjected to flash chromatography (1:2 v/v EtOAc/hexane elution). Concentration of the appropriate fractions $\left(\mathrm{R}_{f} 0.3,1: 1\right.$ v/v EtOAc/hexane) then gave the ent-KDN derivative 21 (4.6 mg, $42 \%$ over 3 steps) as a colorless solid, mp 100-102 ${ }^{\circ} \mathrm{C}\left(\right.$ lit. $\left.^{5} \mathrm{mp} 100-104^{\circ} \mathrm{C}\right) ;[\alpha]_{\mathrm{D}}^{18}+26.2\left(c 0.18, \mathrm{CHCl}_{3}\right) ;{ }^{1} \mathrm{H}$ NMR $\left(500 \mathrm{MHz}, \mathrm{CDCl}_{3}\right)$ ठ 5.39 (1H, dd, H7, J 6.3, 2.4), 5.26 (1H, ddd, H4, J 11.7, 9.8, 5.4), 5.15 (1H, a-dt, H8, J 5.9, 2.4), 4.97 (1H, a-t, H5, J 9.8), 4.43 (1H, dd, H9, J 12.7, 2.4), 4.18 (1H, dd, H6, J 10.3, 2.4), 4.14 (1H, dd, H9', J 12.7, 5.9), 3.79 (3H, s, OMe), $2.62(1 \mathrm{H}, \mathrm{dd}, \mathrm{H} 3, J$ 13.7, $5.4 \mathrm{~Hz}), 2.15\left(3 \mathrm{H}, \mathrm{s}, \mathrm{CH}_{3} \mathrm{CO}\right), 2.11(3 \mathrm{H}, \mathrm{s}$, $\left.\mathrm{CH}_{3} \mathrm{CO}\right), 2.07$ (3H, s, $\left.\mathrm{CH}_{3} \mathrm{CO}\right), 2.07$ (1H, m, H3', obscured), 2.04 (3H, s, $\left.\mathrm{CH}_{3} \mathrm{CO}\right), 2.02(3 \mathrm{H}, \mathrm{s}$, $\mathrm{CH}_{3} \mathrm{CO}$ ), 2.01 (3H, s, $\left.\mathrm{CH}_{3} \mathrm{CO}\right) ;{ }^{13} \mathrm{C}$ NMR (125.7 MHz, $\mathrm{CDCl}_{3}$ ) $\delta 170.6(\mathrm{C}), 170.1(\mathrm{C}), 170.0(\mathrm{C}), 169.7$ (C), $169.6(\mathrm{C}), 168.2(\mathrm{C}), 166.1(\mathrm{C}), 97.3(\mathrm{C}), 71.4(\mathrm{CH}), 70.2(\mathrm{CH}), 68.7(\mathrm{CH}), 67.3(\mathrm{CH}), 66.8(\mathrm{CH})$, 
$61.8\left(\mathrm{CH}_{2}\right), 53.2\left(\mathrm{CH}_{3}\right), 35.4\left(\mathrm{CH}_{2}\right), 20.8(3)\left(\mathrm{CH}_{3}\right), 20.7(5)\left(\mathrm{CH}_{3}\right), 20.6\left(\mathrm{CH}_{3}\right)$ (three signals due to acetate methyl groups obscured or overlapping); IR ( $\mathrm{NaCl}$, thin film) 2959, 1749, 1436, 1371, 1224, 1169, 1113, 1052, 1012, 945, $819 \mathrm{~cm}^{-1}$; MS (ESI+) $m / z 557\left(\mathrm{M}+\mathrm{Na}^{+}, 100 \%\right)$; HRS (FAB) $m / z$ calcd for $\mathrm{C}_{22} \mathrm{H}_{30} \mathrm{O}_{15} \mathrm{Cs}\left(\mathrm{M}+\mathrm{Cs}^{+}\right)$: 667.0634. Found: 667.0669.

\section{References}

1. Reddy, K. K.; Saady, M.; Falck, J. R.; Whited, G. J. Org. Chem. 1995, 60, 3385-3390.

2. For some closely related conversions see: (a) Shing, T.K.M.; Tang, Y. J. Chem. Soc., Chem. Commun. 1990, 748-749. (b) Shing, T. K. M.; Tang, Y. Tetrahedron 1991, 47, 4571-4578. (c) Alves, C.; Barros, M. T.; Maycock, C. D.; Ventura, M. R. Tetrahedron 1999, 55, 8443-8456.

3. Bianco, A.; Brufani, M.; Manna, F.; Melchioni, C. Carbohydrate Res. 2001, 332, 23-31.

4. Hiroya, K.; Ogasawara, K. Chem. Commun. 1998, 2033-2034.

5. Banwell, M.; De Savi, C.; Watson, K. Chem. Commun. 1998, 1189-1190. 
nlh177f4pptCDC13H1-30/10/03

Pulse Sequence: s2pul

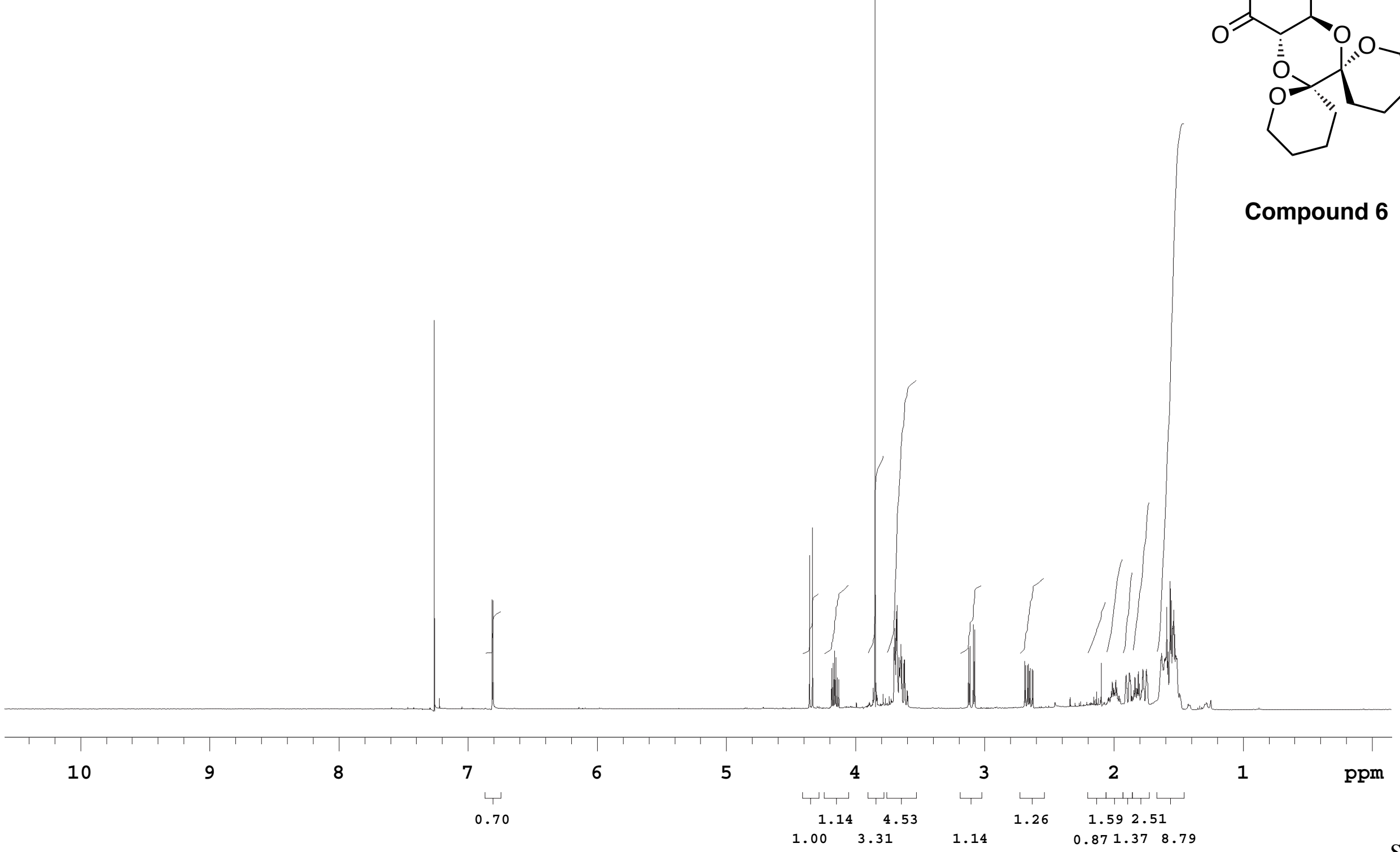


nlh177f4pptCDC13c13

Pulse Sequence: s2pul

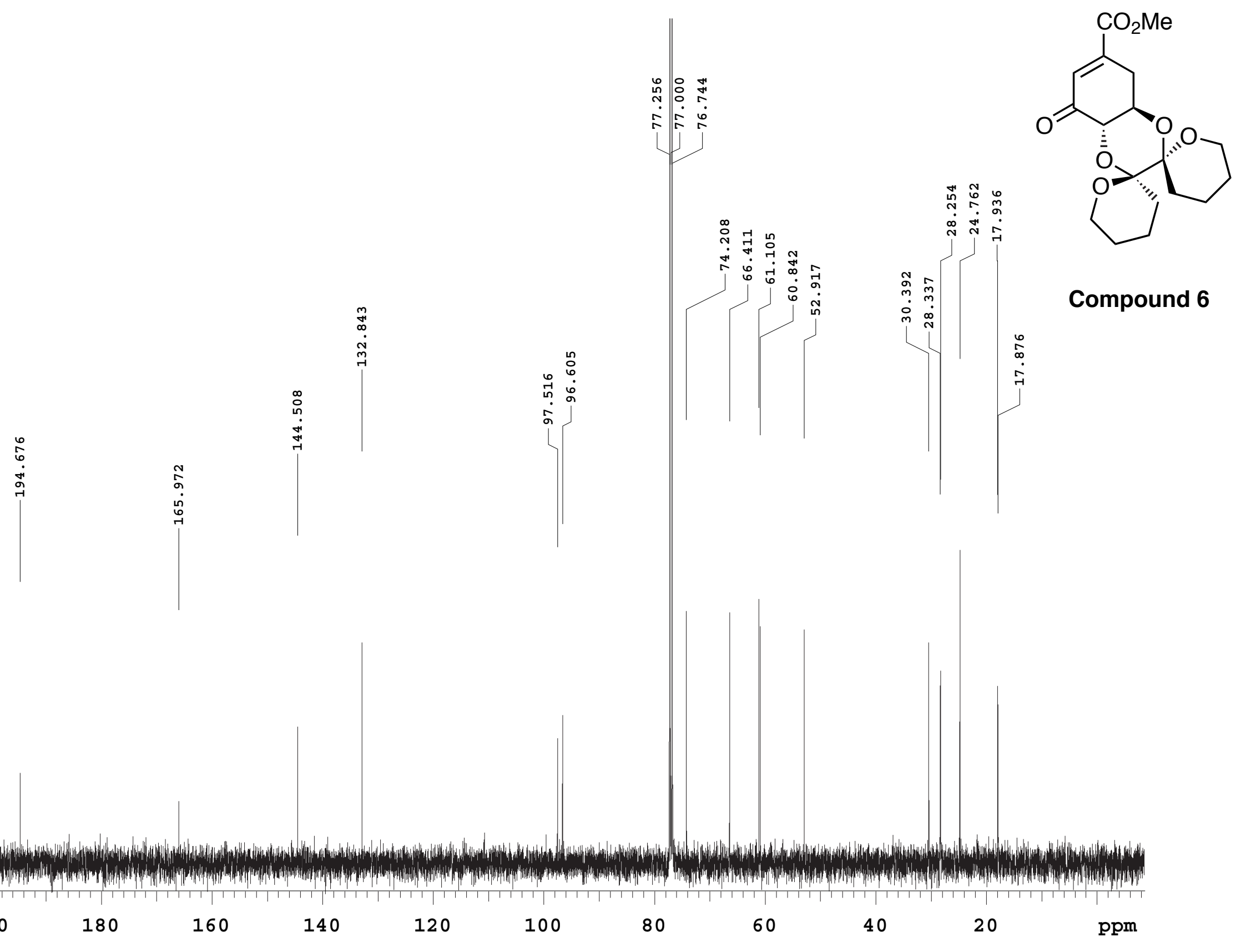


nlh164f2pptCD3ODH1

Pulse Sequence: s2pul

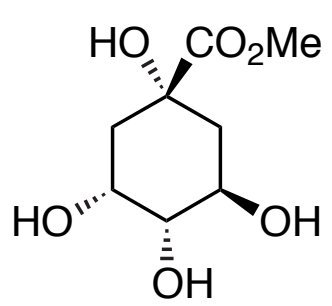

Compound 8

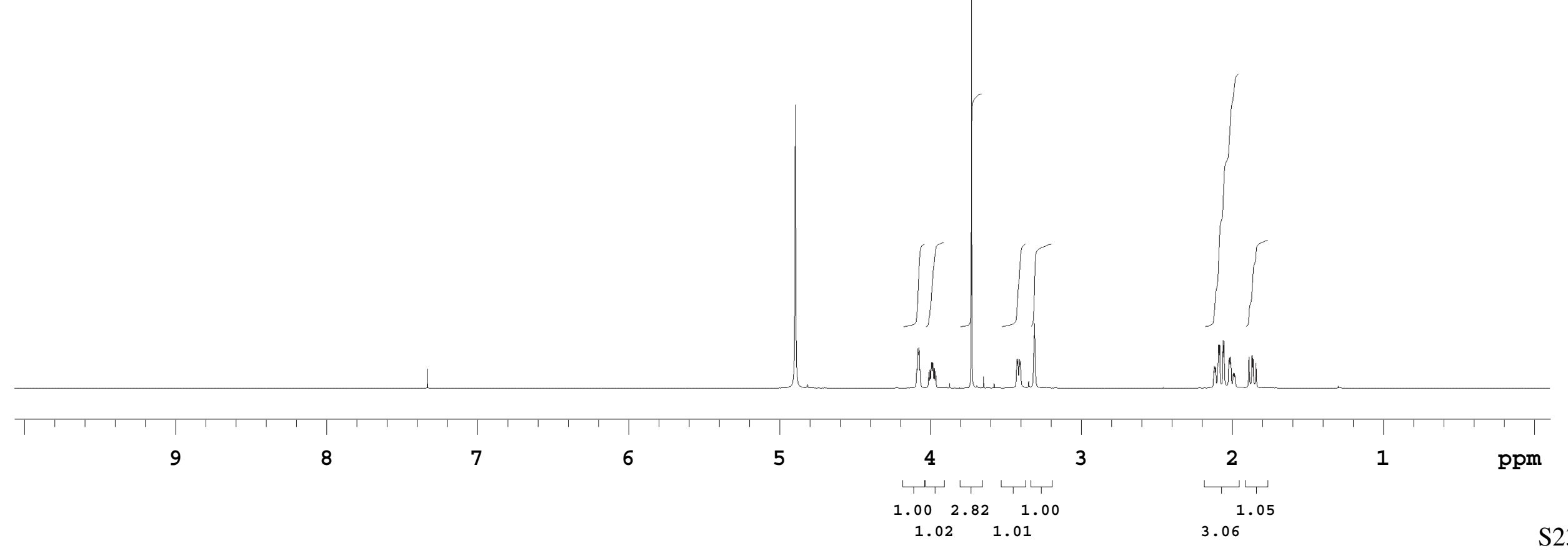


nlh164f2pptCD3ODc13

Pulse Sequence: s2pul

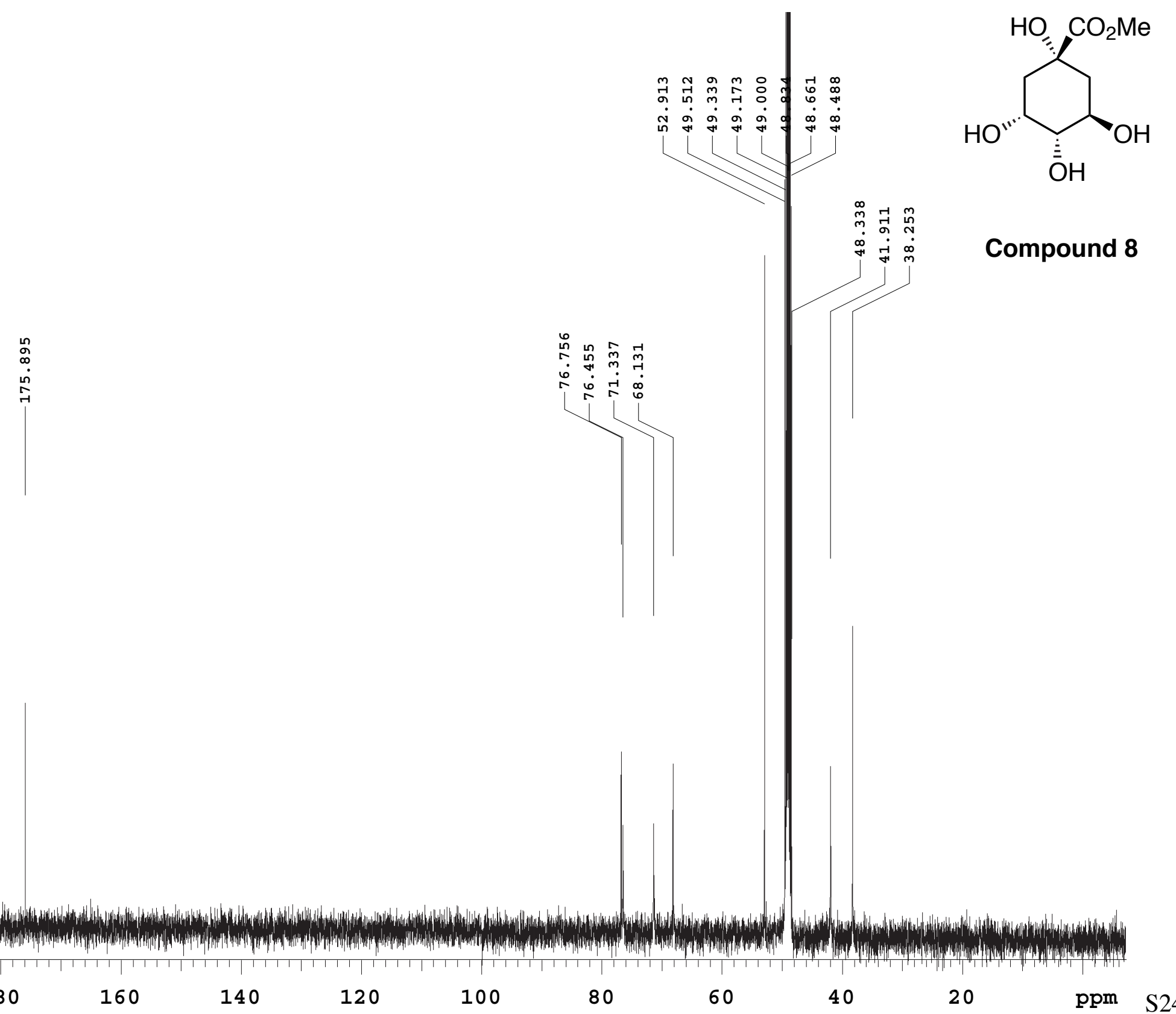


nlh166f37-49CDCl3H1

Pulse Sequence: s2pul

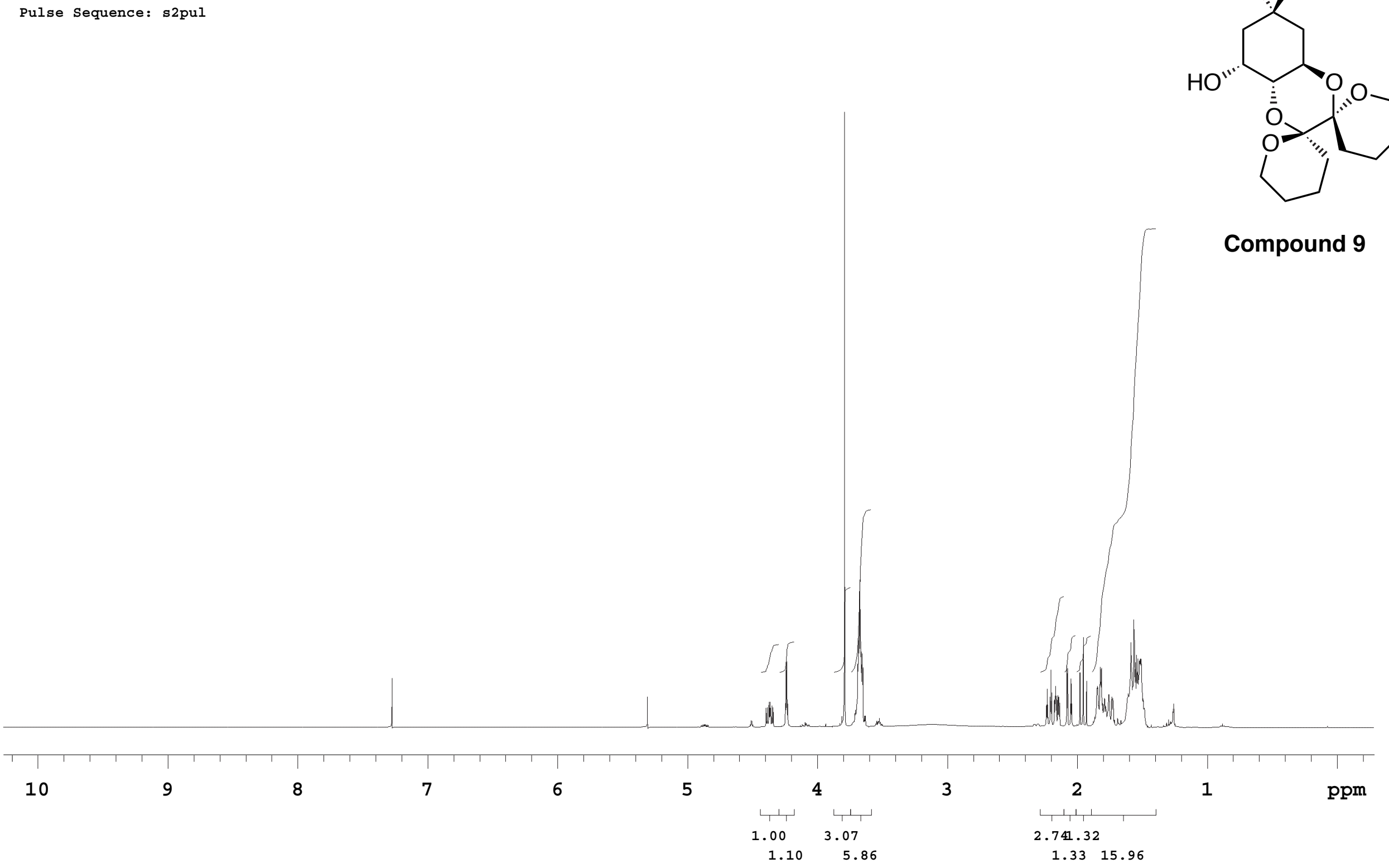


nlh166f37-49CDC13C13nt256

Pulse Sequence: s2pul
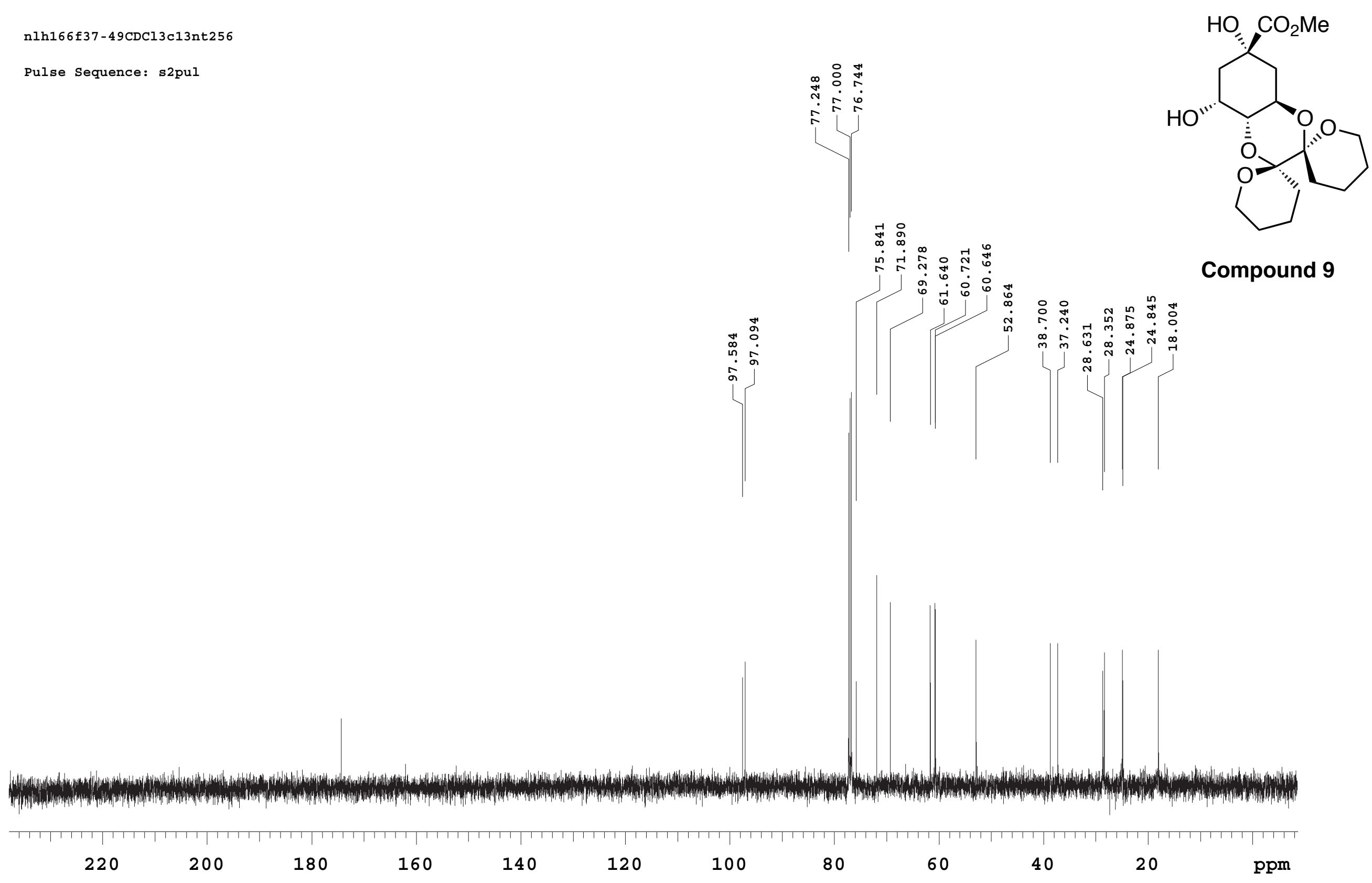
nlh138f7-24CDCl3H1 $300 \mathrm{MHz}$

Pulse Sequence: s2pul

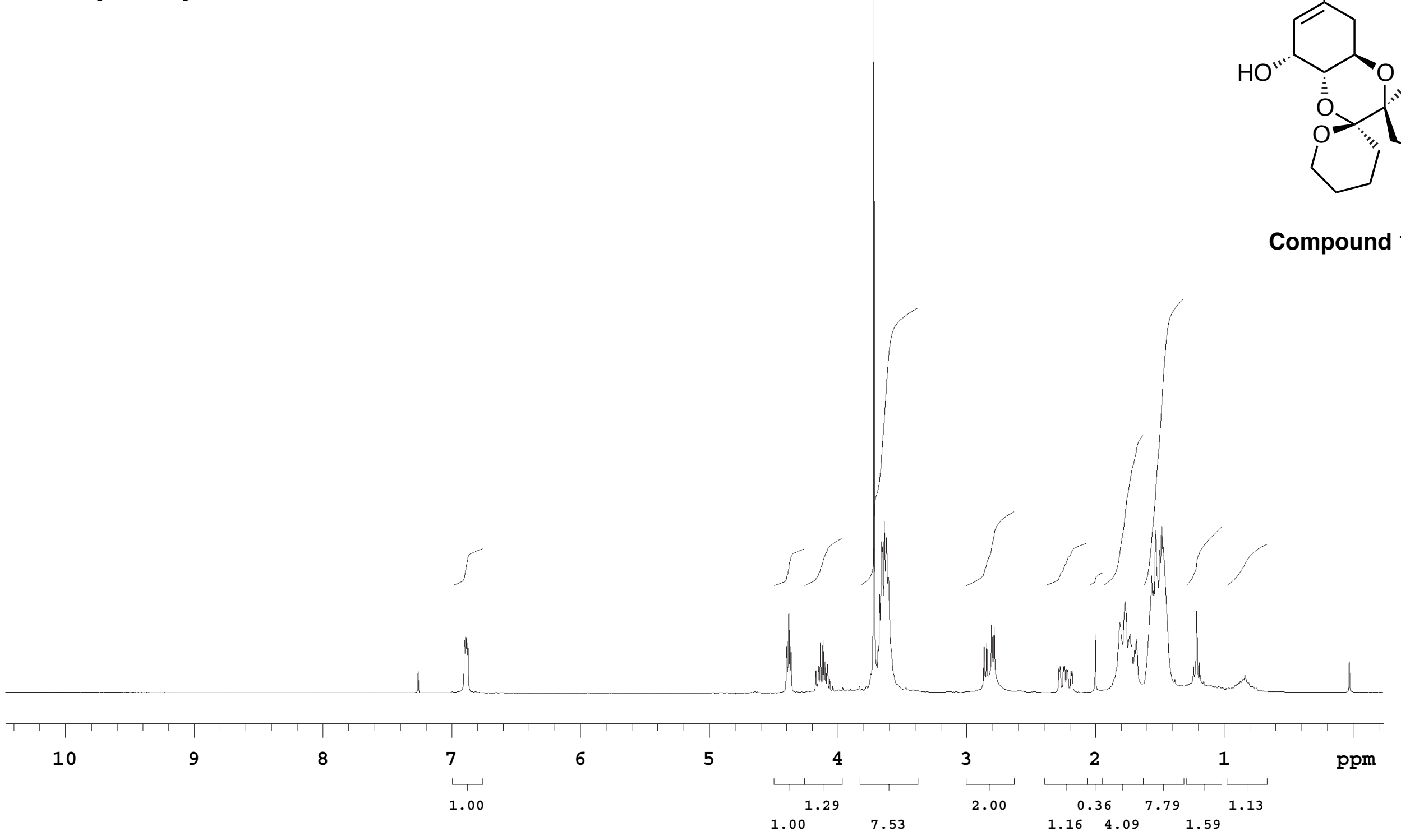


nlh138f7-24CDC13C13 $300 \mathrm{MHz}$

Pulse Sequence: s2pul

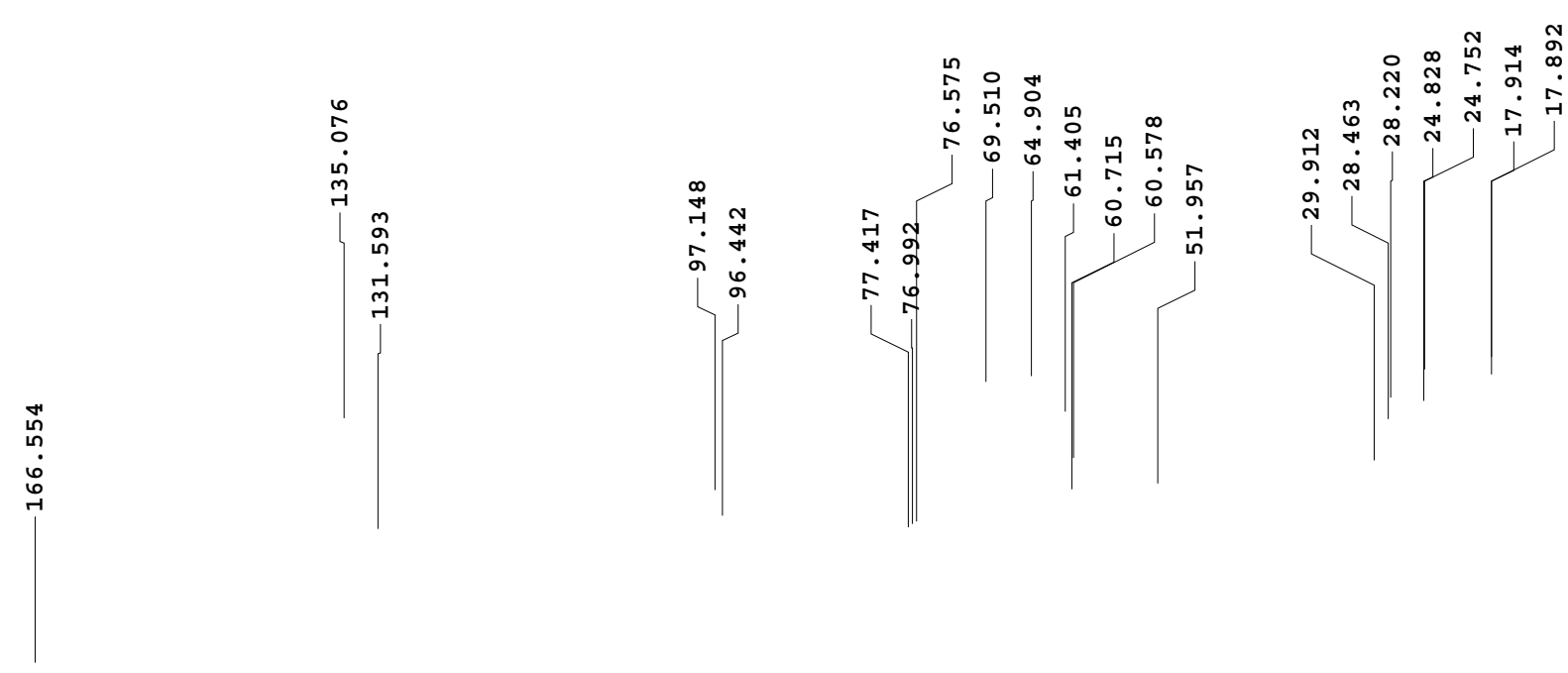

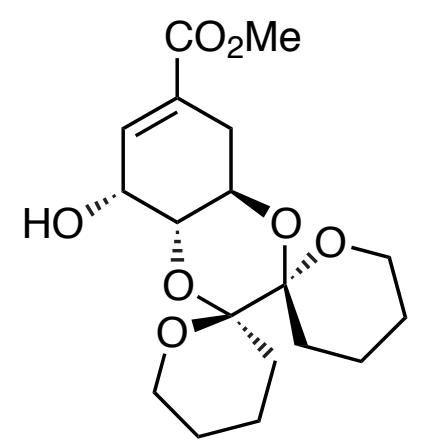

Compound 11

wintw

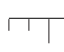

220

200

180

160

140

120

100

80

60

40

20

0

ppm 
Archive directory: /export/home/mercury/vnmrsys/data

Sample directory: auto50_CustomQ_29Aug2001

Pulse Sequence: s2pul

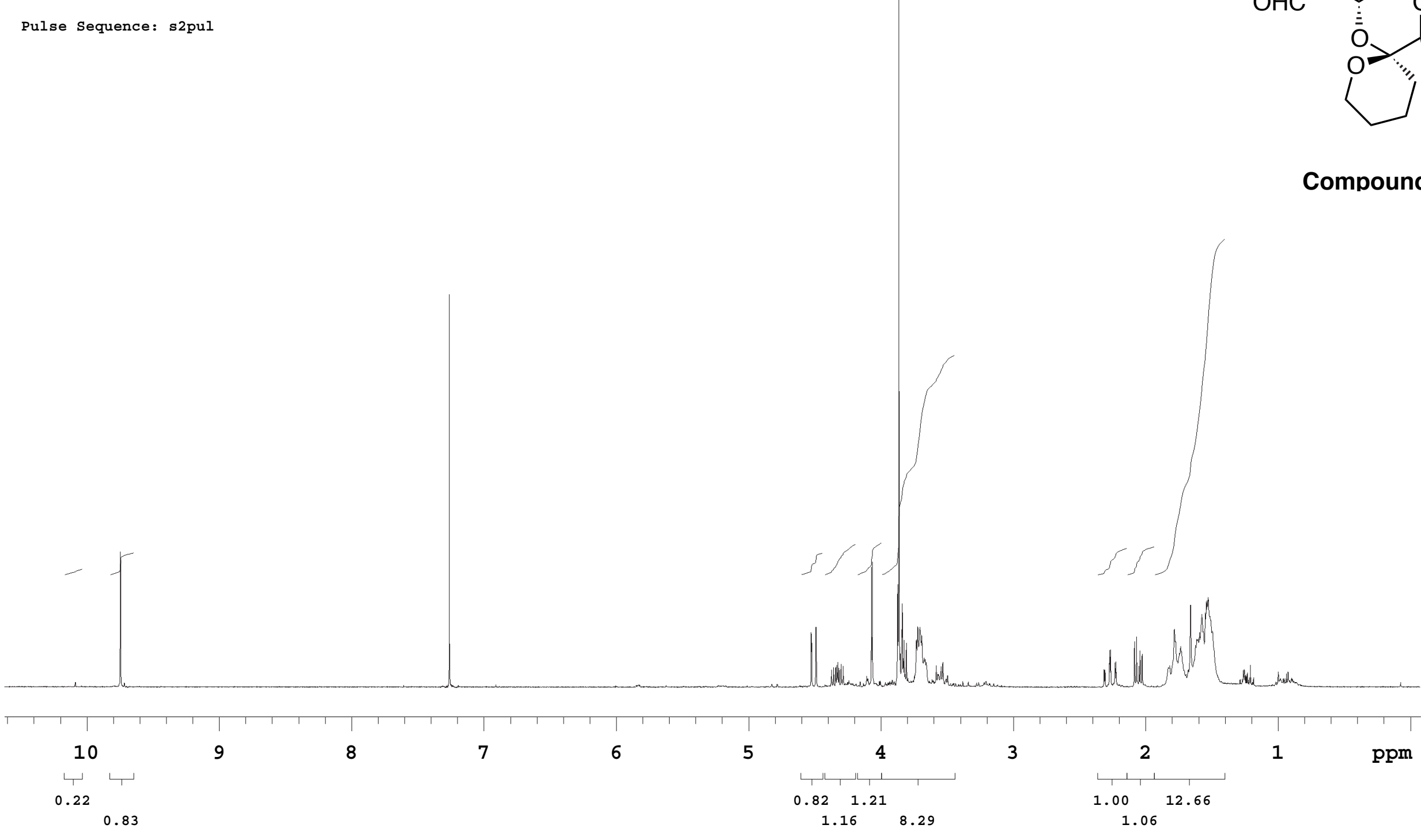


kj $4-44-1-03-13 C$

Archive directory: /export/home/mercury/vnmrsys/data Sample directory: auto50_CustomQ_29Aug2001

Pulse Sequence: s2pul

$\stackrel{\circ}{0}$
$\stackrel{0}{\circ}$
ఫे

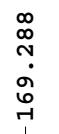

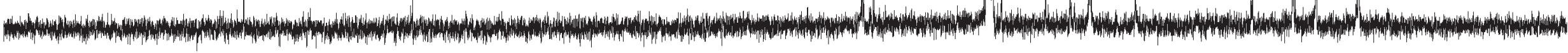




\section{$\mathrm{kj} 6 \mathrm{de} 10-1 \mathrm{H} 300$}

Archive directory: /export/home/mercury/vnmrsys/data Sample directory:

Pulse Sequence: s2pul

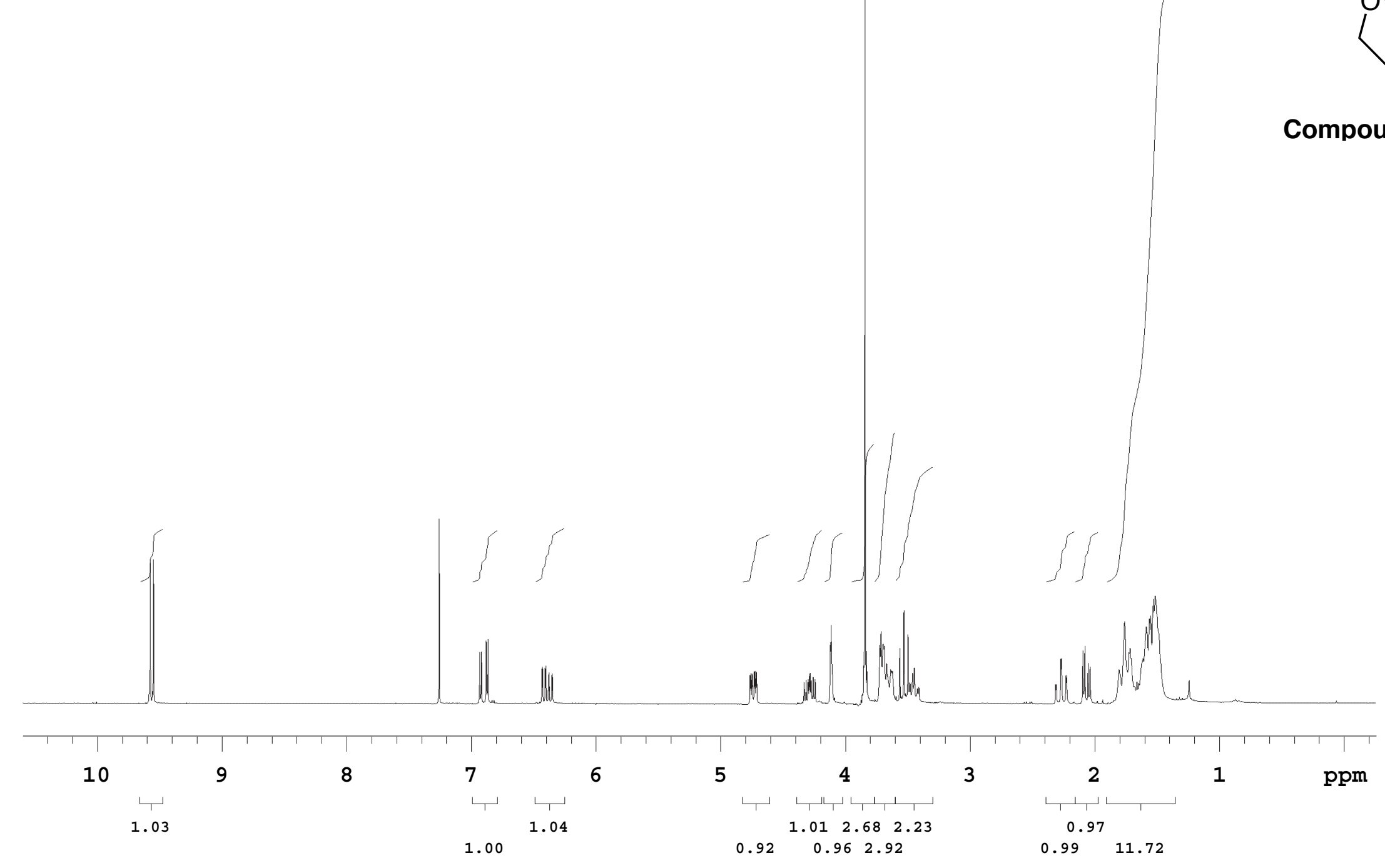




\section{kj7de10-13C_300}

Archive directory: /export/home/mercury/vnmrsys/data Sample directory:

Pulse Sequence: s2pul

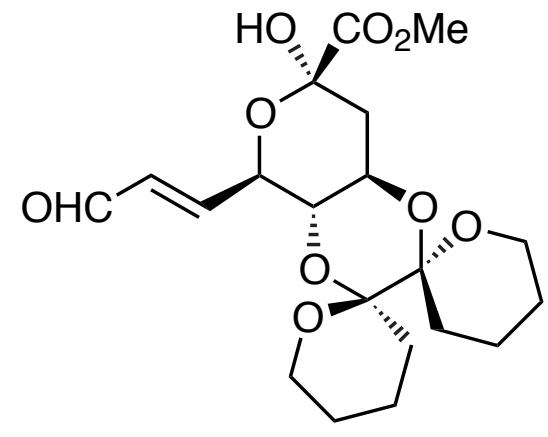

Compound 13

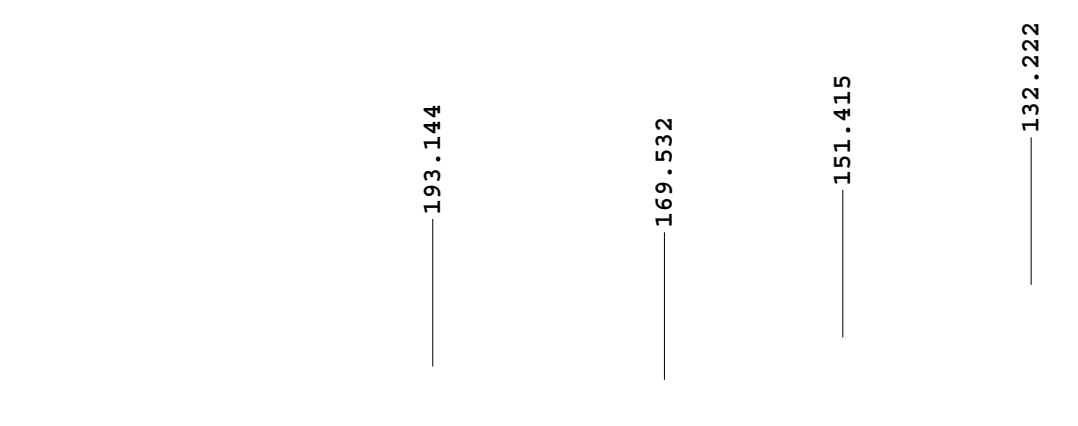

กิำ

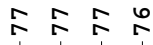

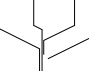

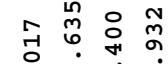

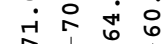

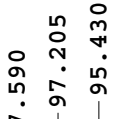

?ै ลั
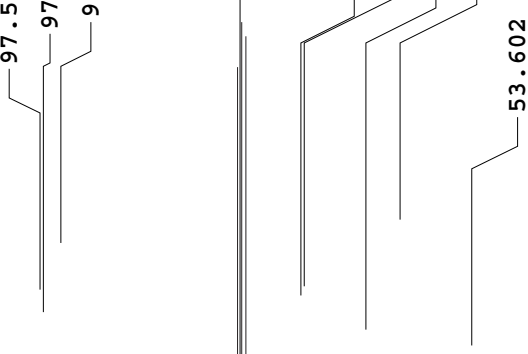

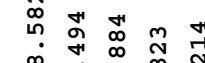

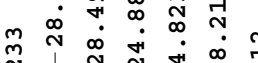

กิ

winn 
Archive directory: /export/home/mercury/vnmrsys/data Sample directory:

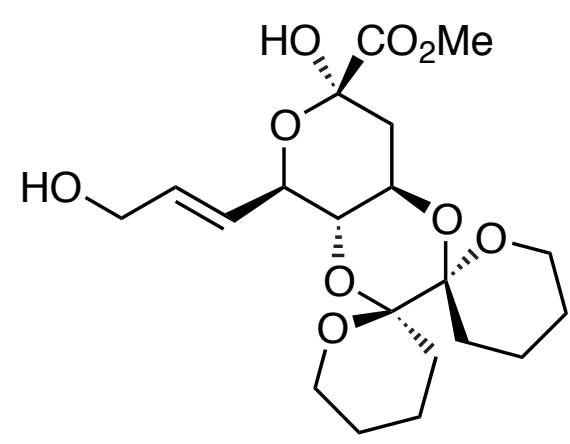

Compound 14

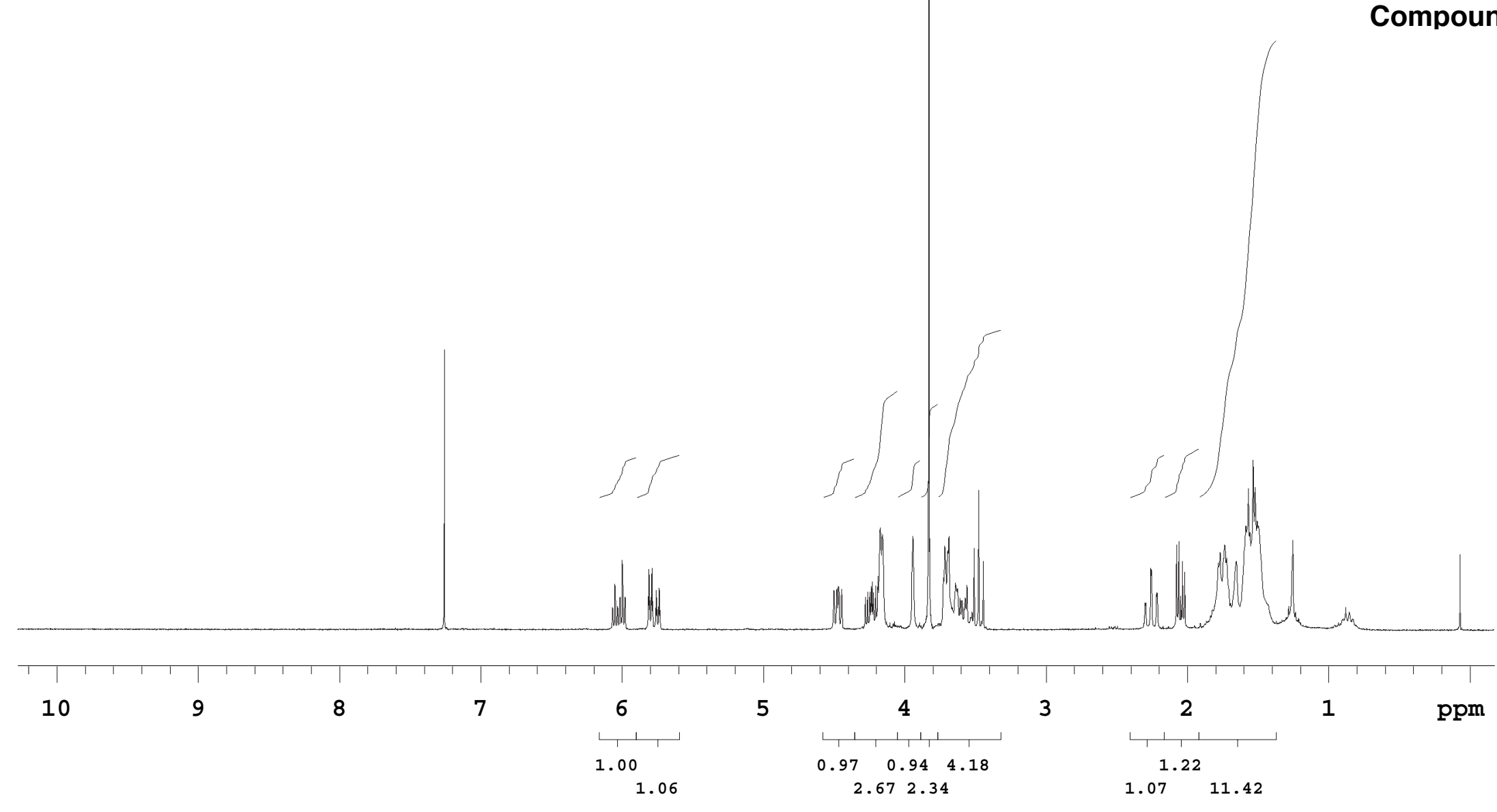


nlh21f22-29CDC13c13

Archive directory: /export/home/mercury/vnmrsys/data Sample directory:

Pulse Sequence: s2pul

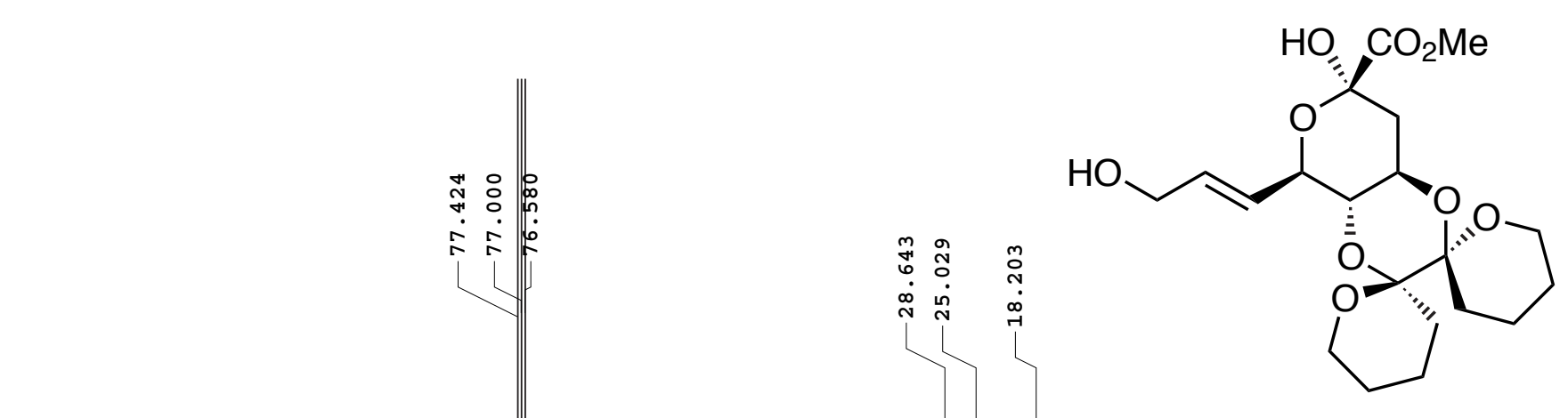

ڤ̊

苟

$\dot{m}$

m

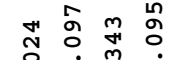

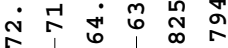

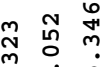

ลे ลू

L.]

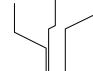

Compound 14

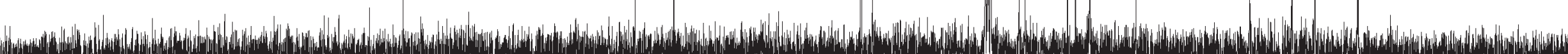

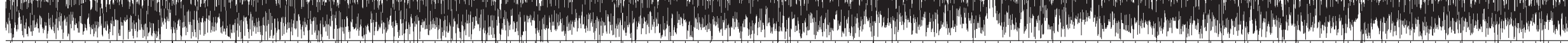




\section{nlh145c2f13-20CD3ODh1}

Pulse Sequence: s2pul

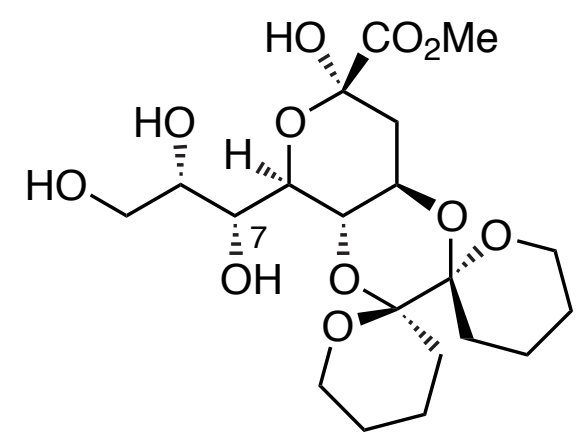

Compound 15

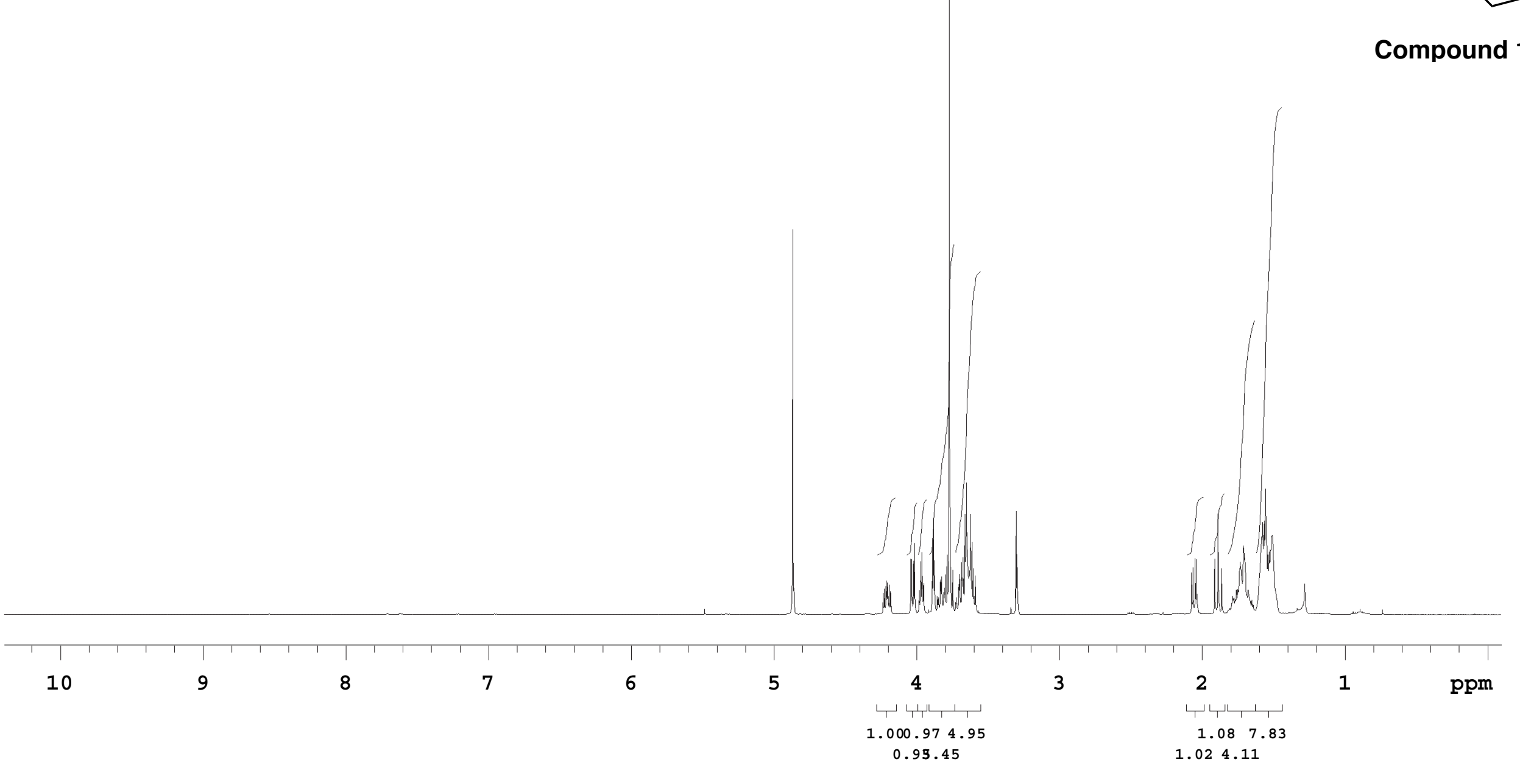


nlh145c2f13-20CD3ODc13

Pulse Sequence: s2pul

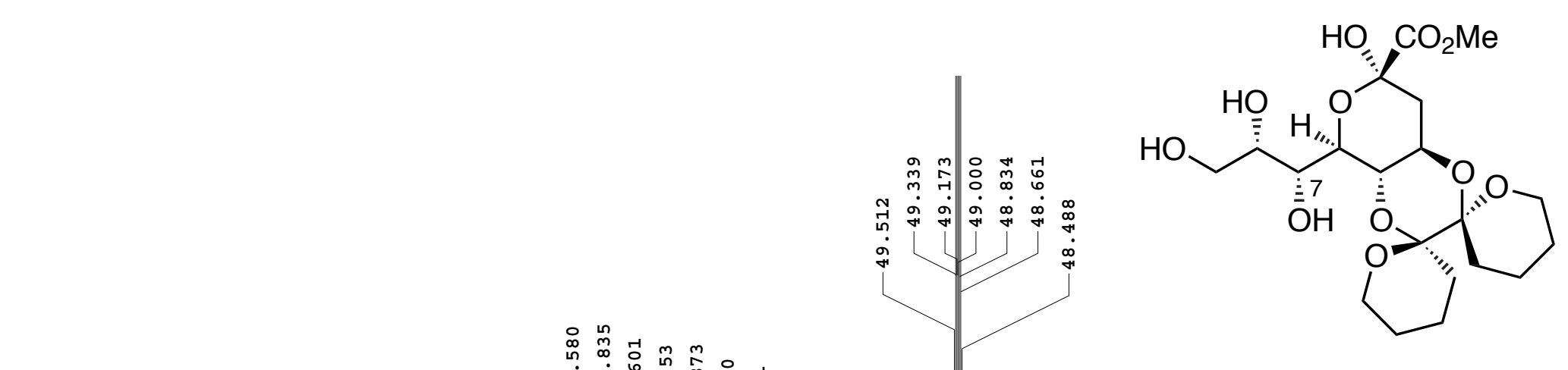

Compound 15

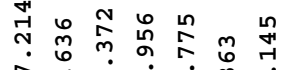

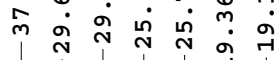

1 ।
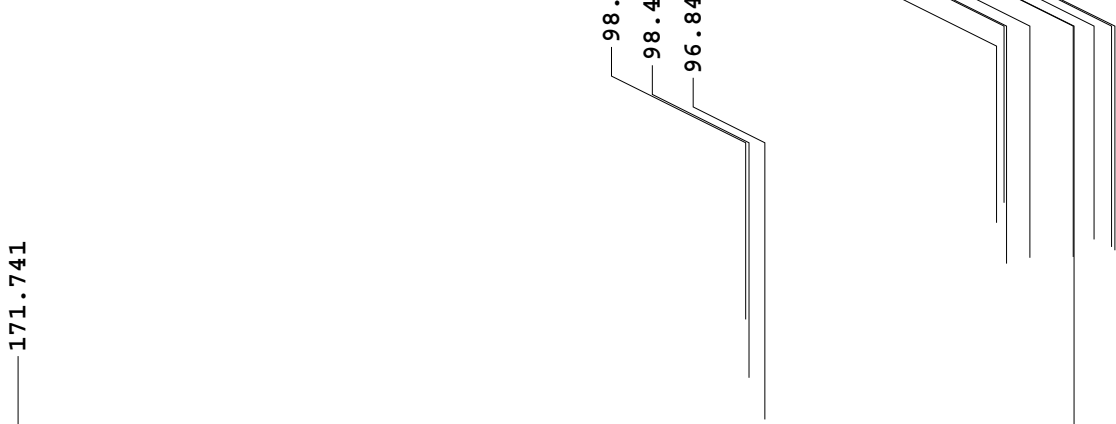
nlh145c2f24-50CD3ODh1

Pulse Sequence: s2pul

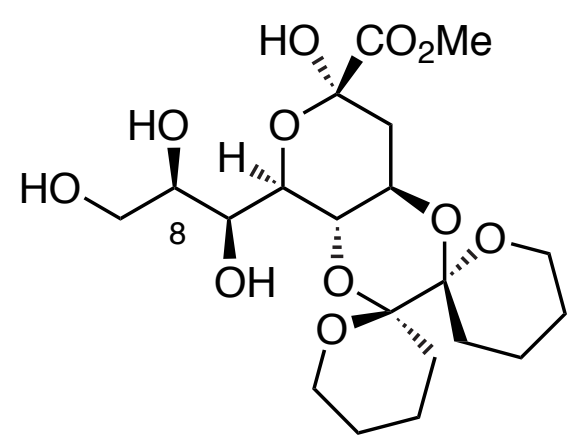

Compound 16

10

9

8

6

1.001 .614 .83

0.82 .95 
nlh145c2f $24-50 C D 30 D c 13$

Pulse Sequence: s2pul

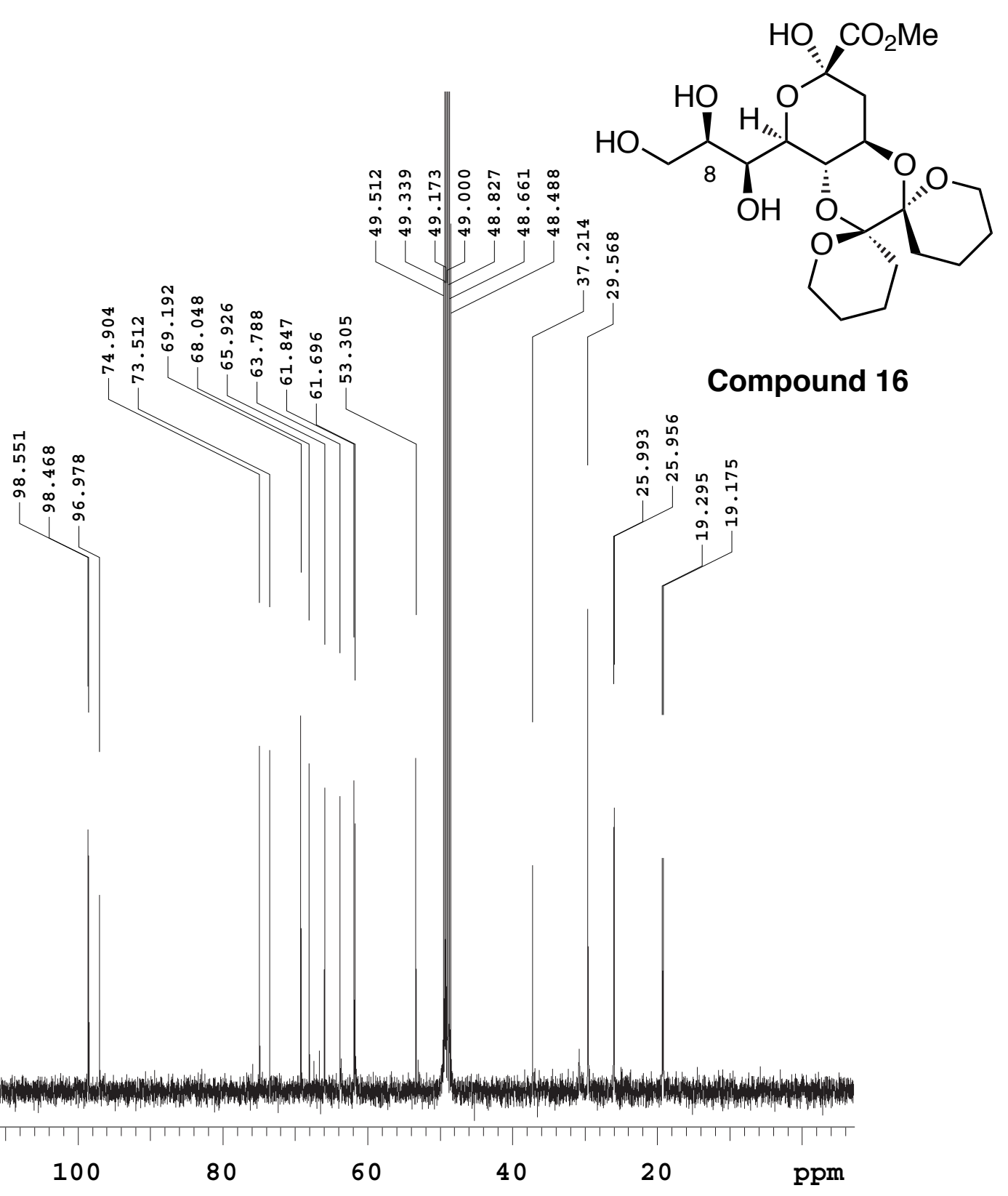




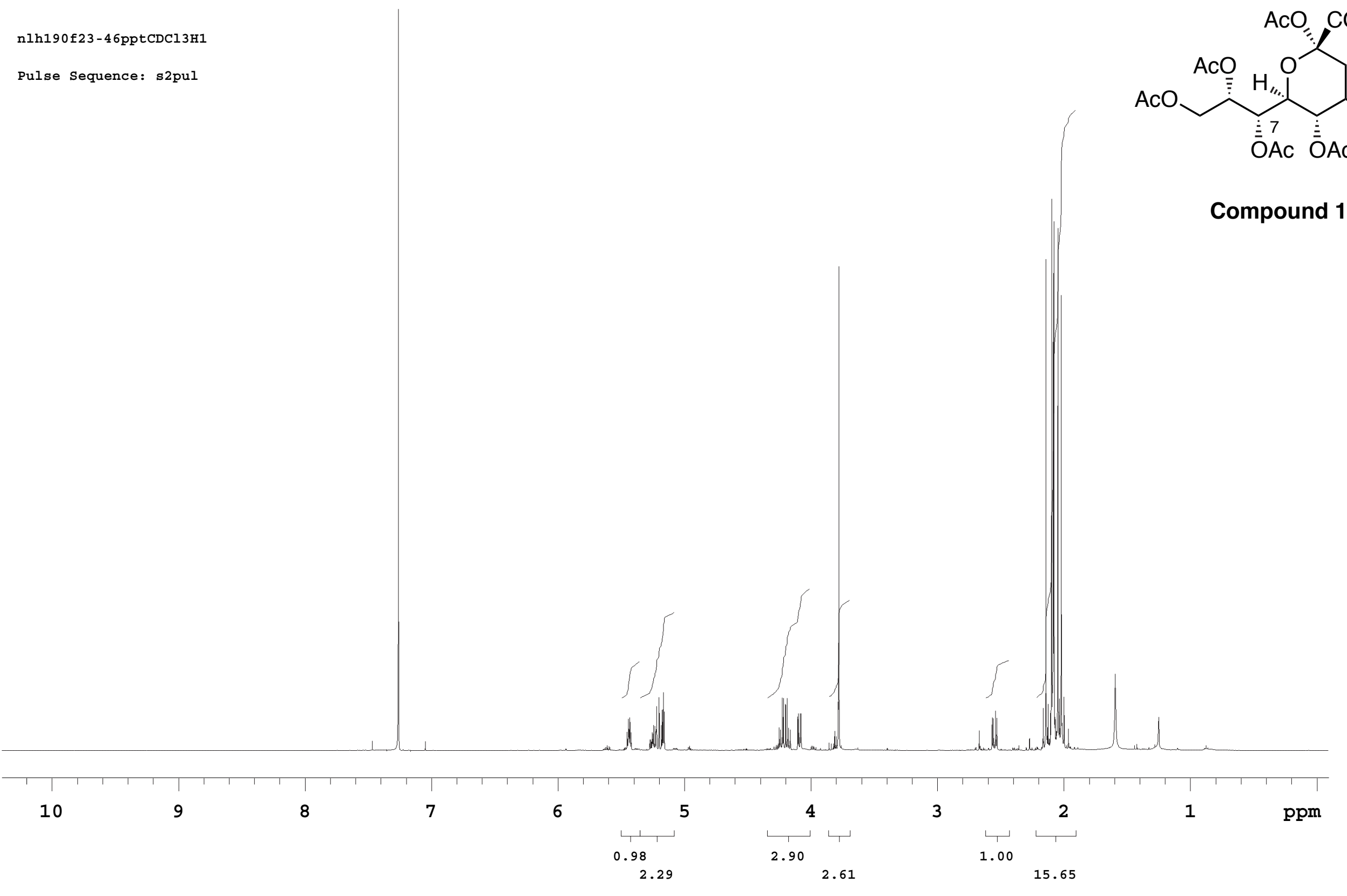


nlh190f23-4 6pptCDC13c13

Pulse Sequence: s2pul

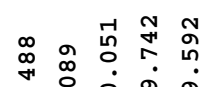

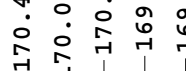

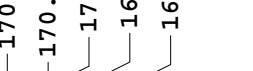

न $\quad \stackrel{\text { กิ }}{\circ}$

$\stackrel{\infty}{\hookrightarrow}$
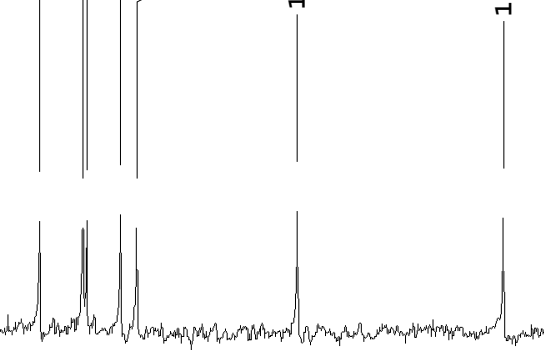

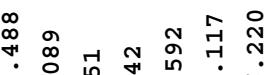

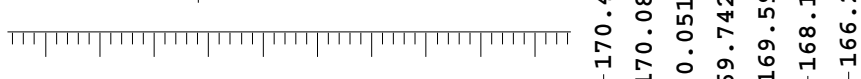
$\begin{array}{llll}170.5 & 169.0 & 167.5 & \mathrm{ppm}\end{array}$
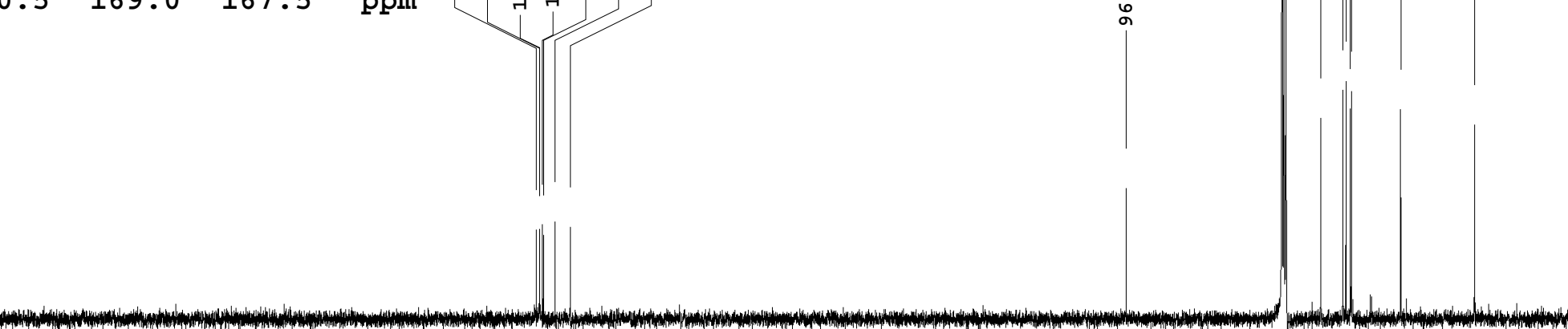

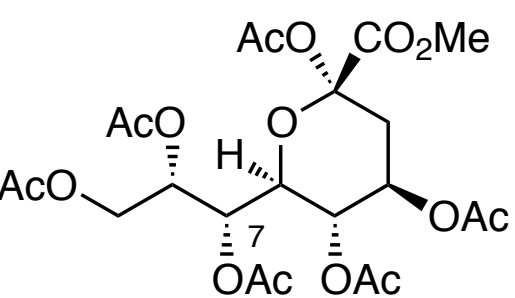

Compound 17

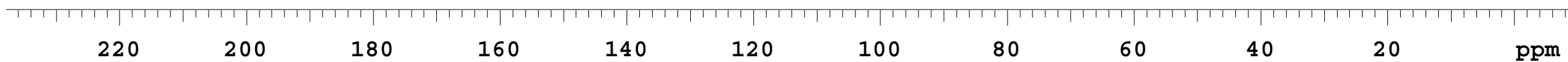


nlh63c3f15-22pptshimCDCl3H1

Pulse Sequence: s2pul

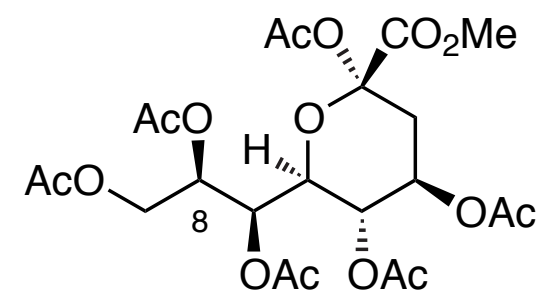

Compound 18

7

6

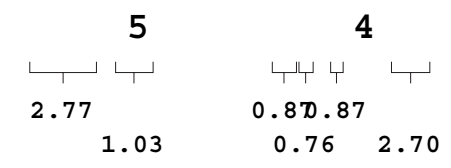

3

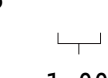

2

16.52 
nlh63c3f15-22pptCDC13c13

Pulse Sequence: s2pul

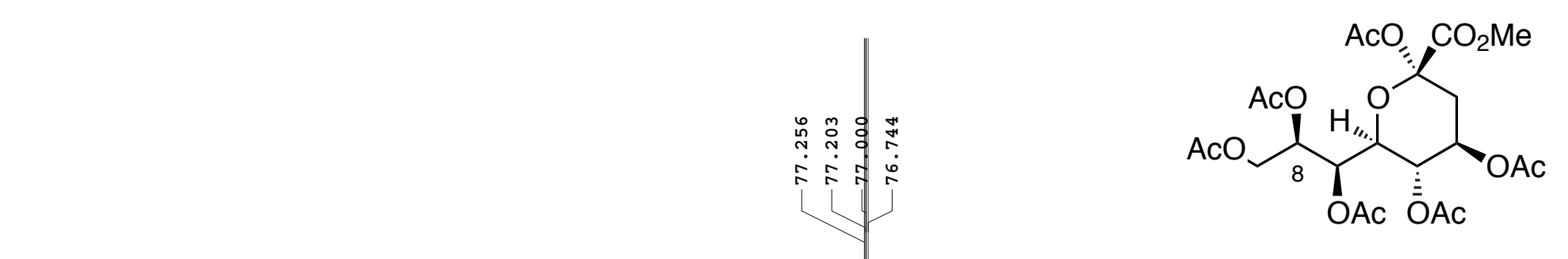

Compound 18
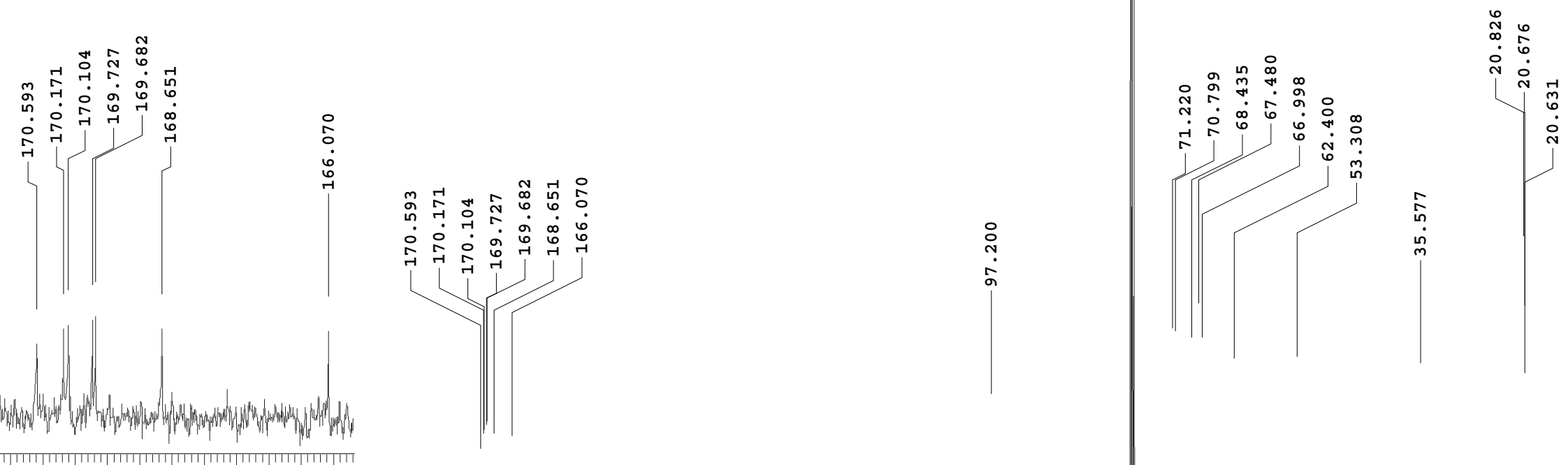

$170.0 \quad 168.0 \quad \mathrm{ppm}$

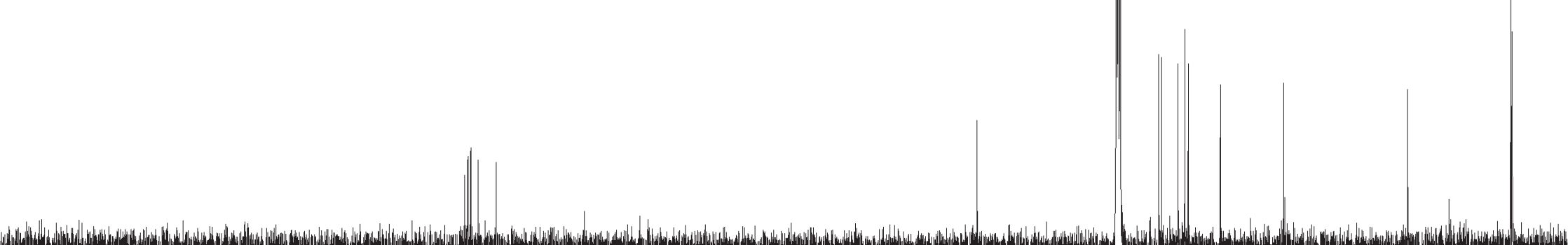

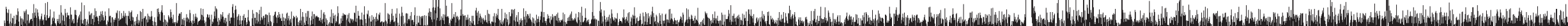

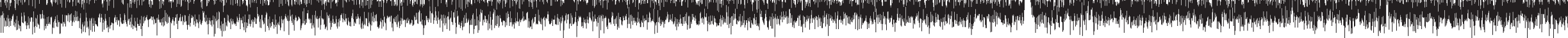


nlh174f34-48C6D6H1 $300 \mathrm{MHz}$

Pulse Sequence: s2pul

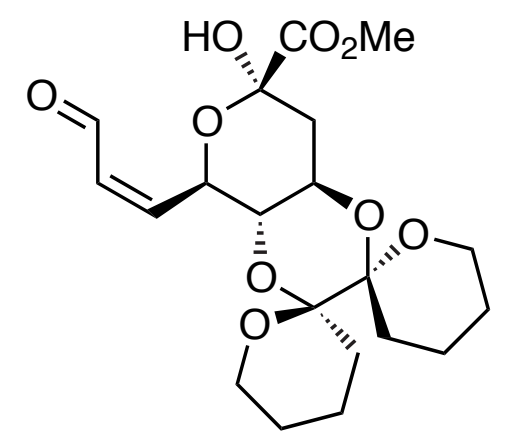

Compound 19

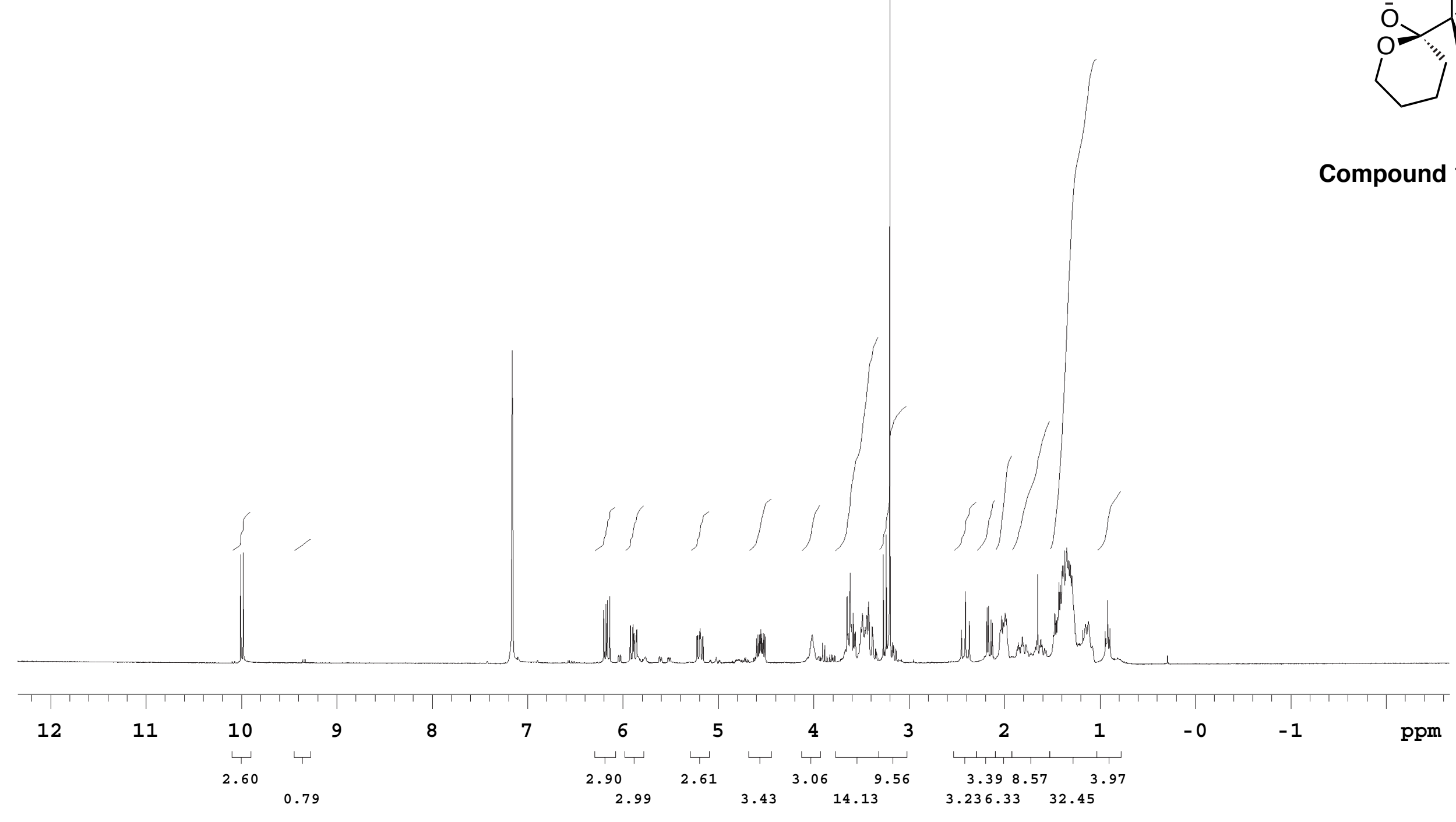


nlh174f34-38C6D6c13 - $300 \mathrm{MHz}$

Pulse Sequence: s2pul
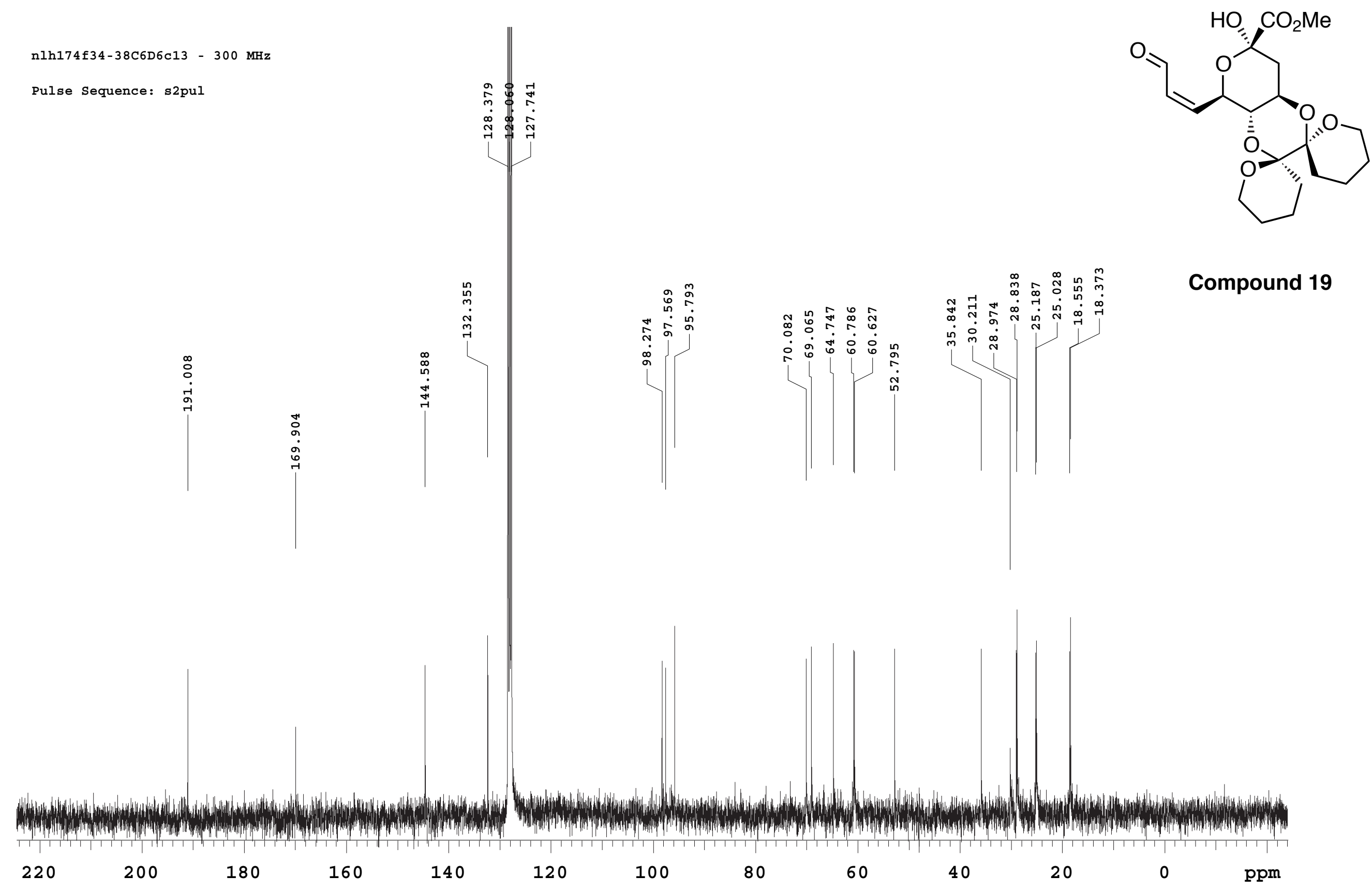
nlh175f18-24CDCl3H1 - $300 \mathrm{MHz}$

Pulse Sequence: s2pul

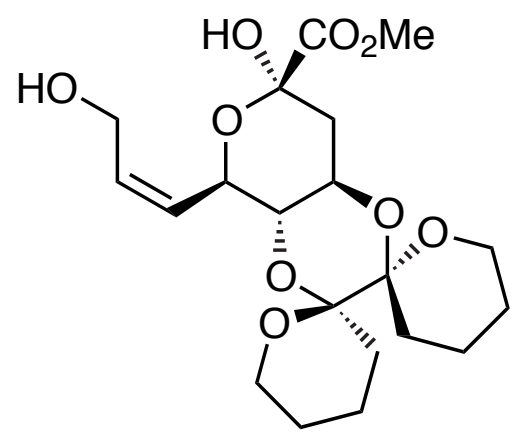

Allylic Alcohol Derived from Compound 19

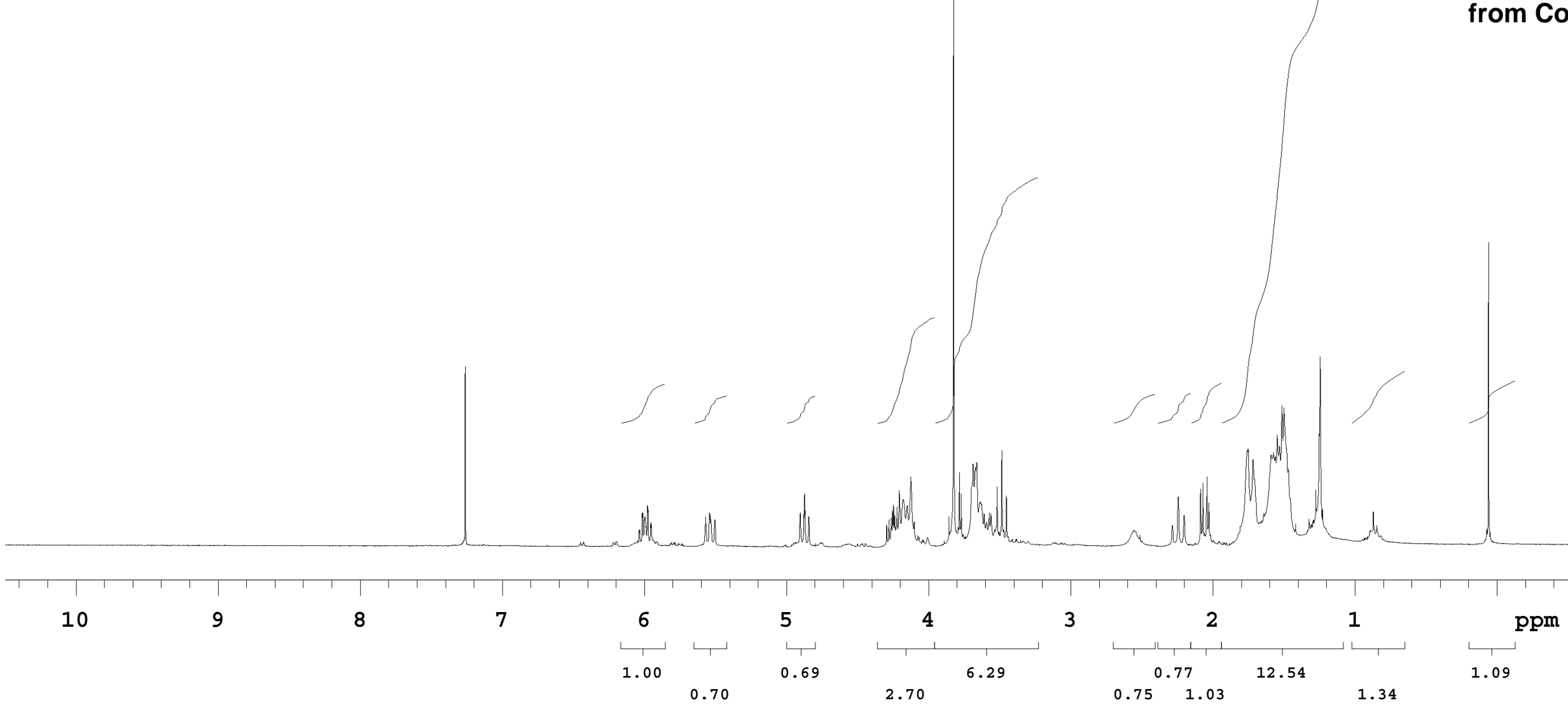


nlh175f18-24CDCl3c13-15/10/03 $300 \mathrm{MHz}$

Pulse Sequence: s2pul

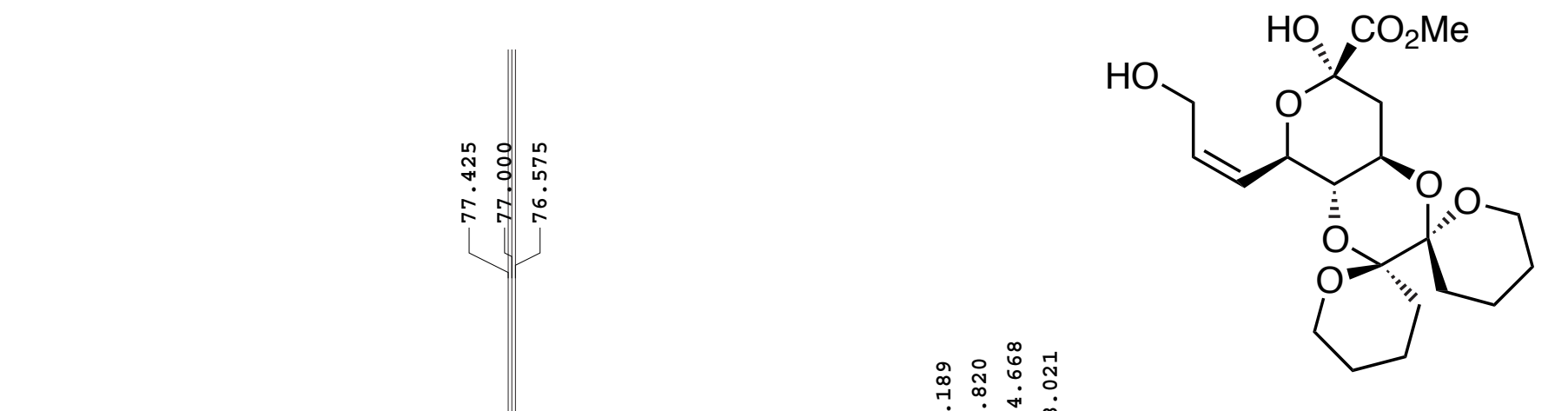

Allylic Alcohol Derived from Compound 19
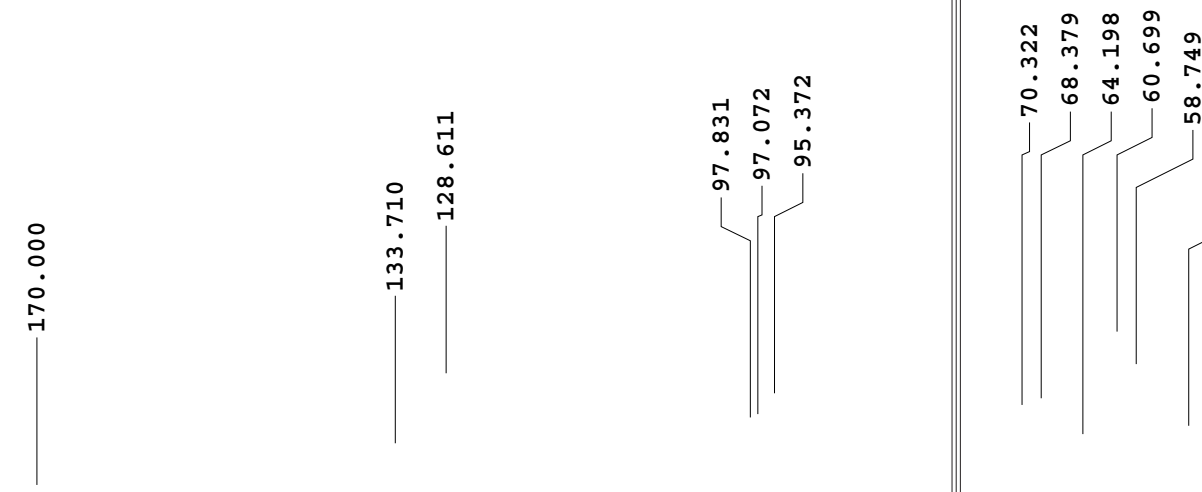
nlh176c2f6c3f11-27CD3ODH1500

Pulse Sequence: s2pul

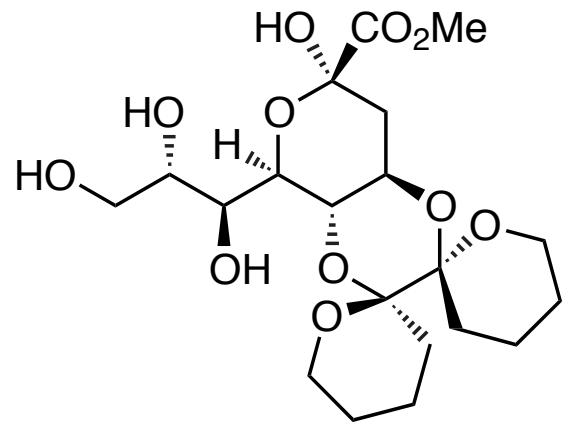

Compound 20

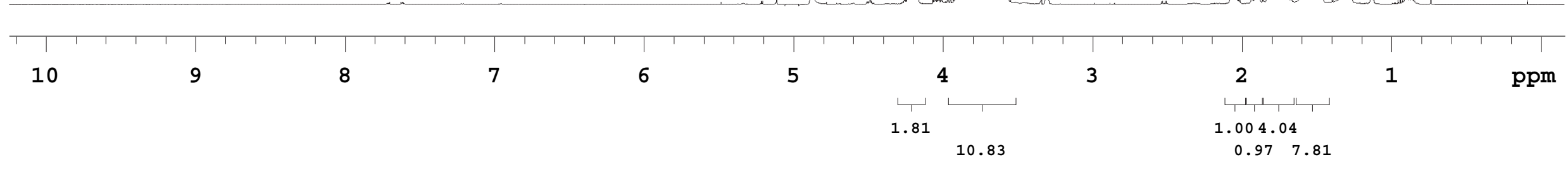




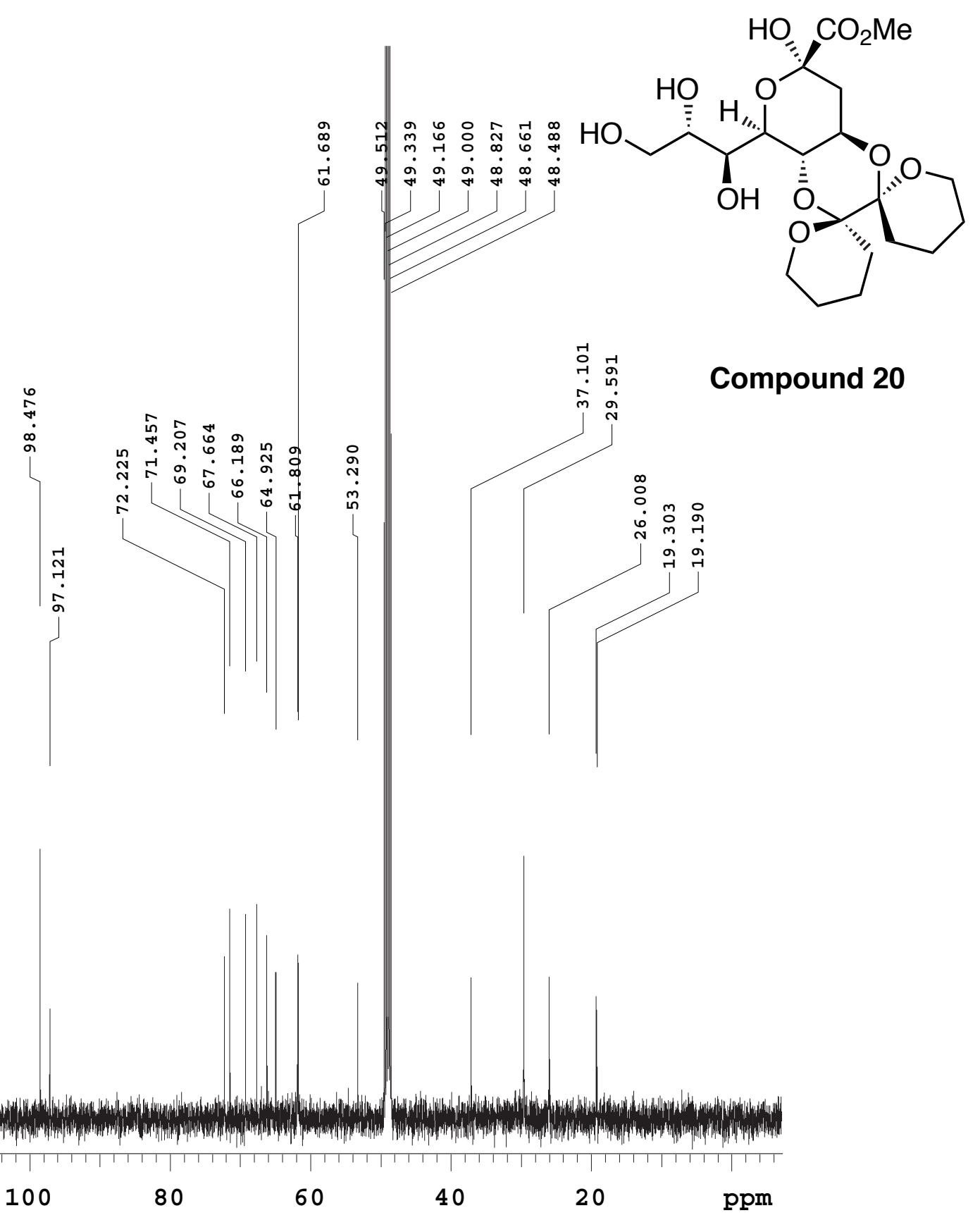


nlh180f15-22pptCDC13H1

Pulse Sequence: s2pul

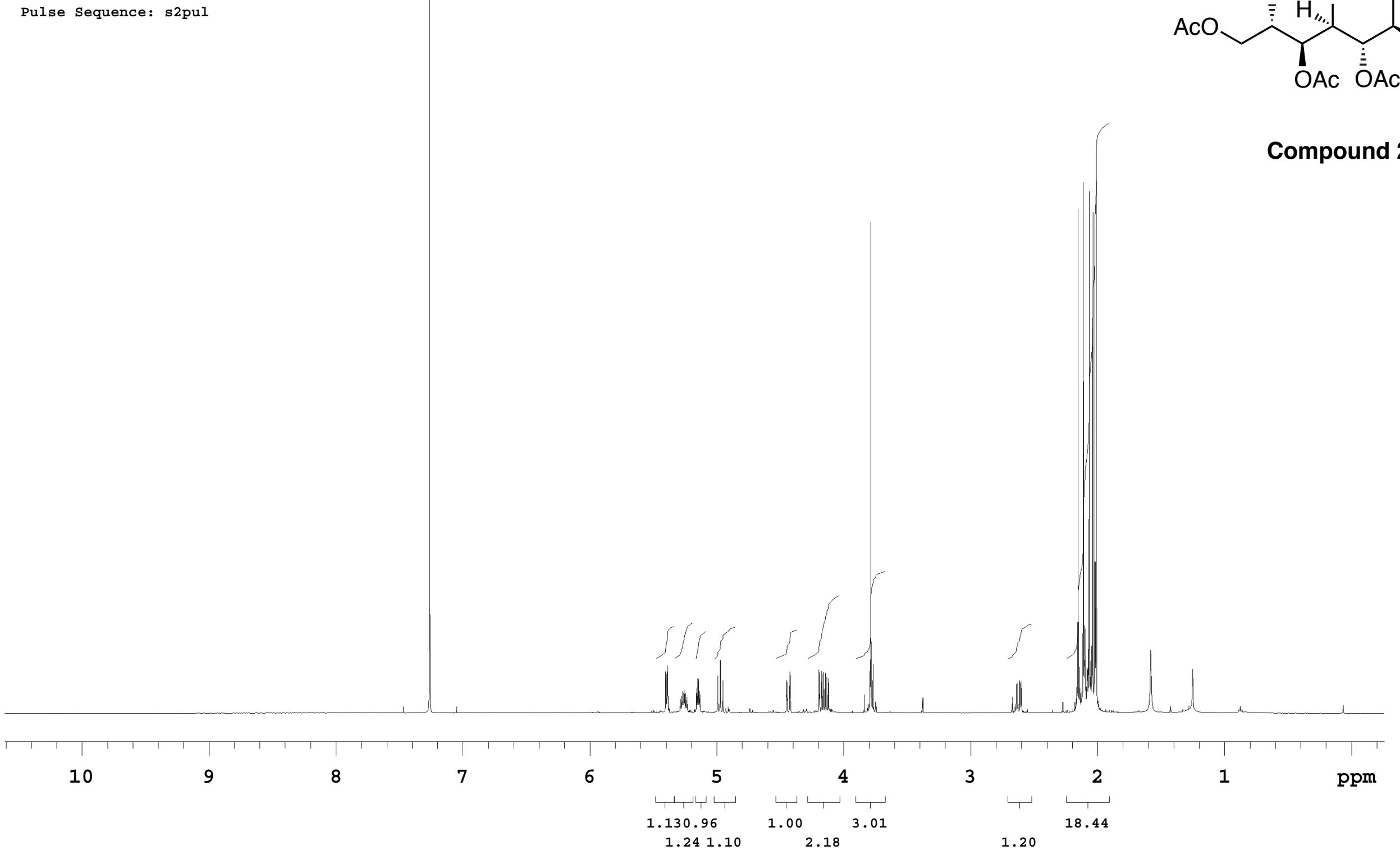


nlh180f15-22pptCDC13c13

Pulse Sequence: s2pul

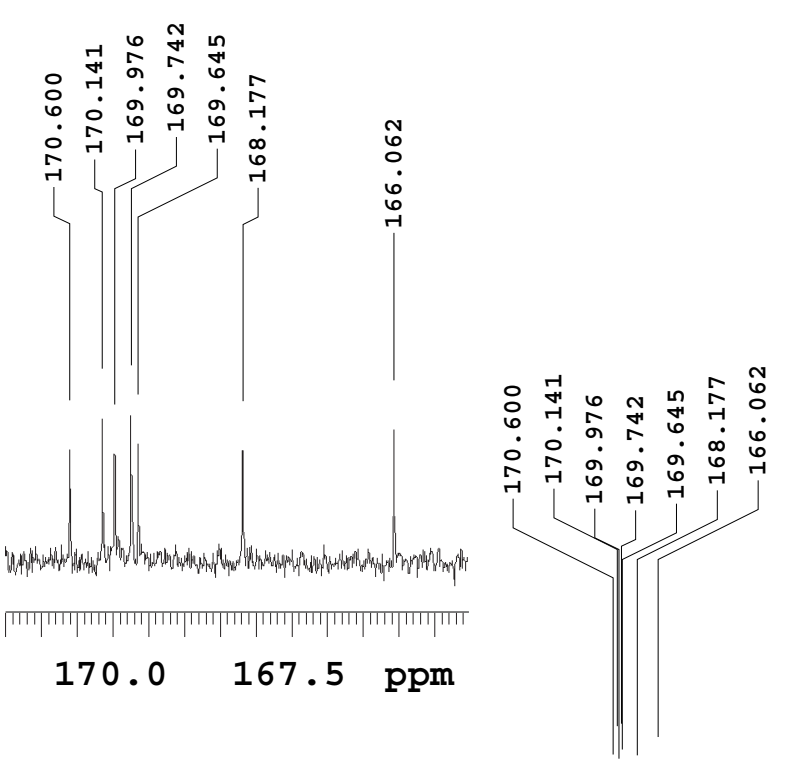

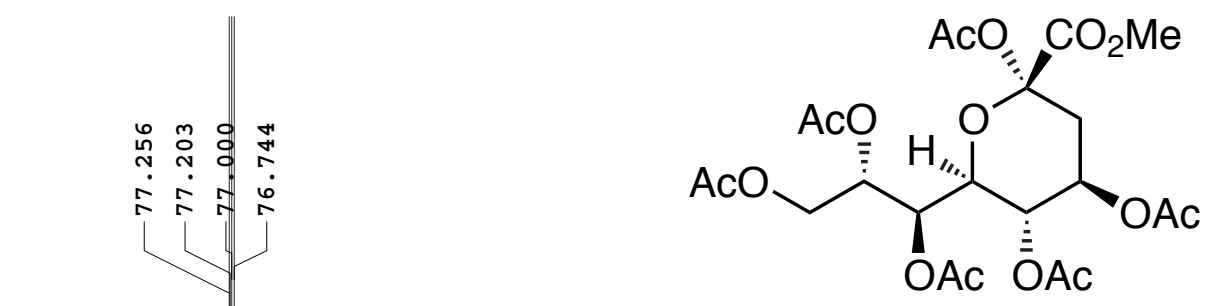

Compound 21

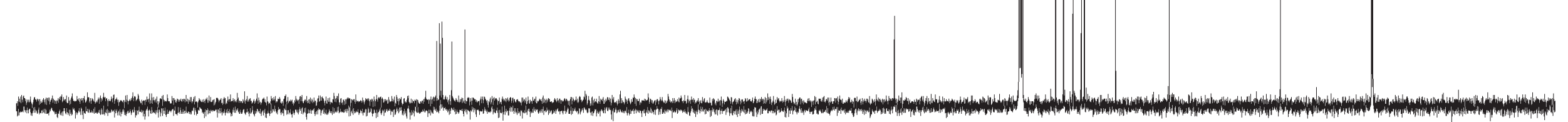

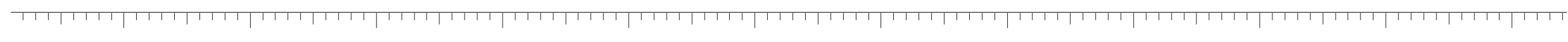

